\title{
A delayed feedback control for network of open canals
}

\author{
Mouhamadou Samsidy Goudiaby • Abdou Sene • \\ Gunilla Kreiss
}

Received: 29 April 2013 / Revised: 2 July 2013 / Accepted: 30 August 2013 / Published online: 23 September 2013

(C) Springer-Verlag Berlin Heidelberg 2013

\begin{abstract}
This paper proposes an algebraic method to design time-delay boundary feedback controllers to regulate the water flow and level in a network of open canals. The network is modeled as $n$ canals with one junction where all canals come together. The Saint-Venant equations are linearized around a prescribed steady state. We consider steady subcritical flow condition and an energy estimate, to build feedback boundary conditions. These boundary conditions depend on data at earlier times and ensure the exponential decrease in $L^{2}$-norm of the solution of the linearized model. A single canal is first treated and afterward the analysis is extended to the network. Finally, the controllers are applied numerically to the nonlinear Saint-Venant equations.
\end{abstract}

Keywords St. Venant equations - Hyperbolic systems . Stabilization $\cdot$ Numerical resolution

Mathematics Subject Classification 35L65 - 35L50 . 93D21 $65 \mathrm{M} 08$

M. S. Goudiaby $(\varangle) \cdot$ A. Sene

Laboratoire d'Analyse Numérique et d'Informatique (LANI), UFR Sciences Appliquées et Technologie,

Université Gaston Berger, B.P 234,

Saint-Louis, Senegal

e-mail: samsidy@yahoo.fr

A. Sene

e-mail: abdou.sene@ugb.edu.sn

G. Kreiss

Division of Scientific Computing,

Department of Information Technology,

Uppsala University, 75105 Uppsala, Sweden

e-mail: gunilla.kreiss@it.uu.se

\section{Introduction}

The one dimensional Saint-Venant equations are nonlinear partial differential equations established by Barré de Saint Venant in [1]. They are, composed by a mass and momentum balance laws, used to describe the behaviour of the flow of water in open canals and rivers. Hydraulic engineers use Saint-Venant equations as a fundamental tool for solving problems regarding the dynamics of canals and rivers.

For many years, the area of controllability, observability and stabilizability in irrigation canals has been considered in the literature as reported in [2,3]. In [3], the authors give a classification of canal control algorithms and an a comprehensive bibliography in this area. A synthesis has been done by Bastin et al. [4], who give contributions through an interesting bibliography, open problems and mathematical challenges concerning irrigation canals.

By means of several theories, various advanced control methods were investigated. Among them, we have the linear quadratic control method which is developed and studied by Balogun et al. [5], Malaterre [6], Weyer [7] and Chen et al. [8]. Through semi-group approach, Xu et al. [9] proposed an proportional and integral output feedback controller using a linear PDE model around a steady state. Litrico et al. [10] have studied an $H_{\infty}$ control for an irrigation canal. Lyapunov analysis and Riemann invariant are also used (see [11-17]). Another approach can be found in [18].

Complex networks of canals have already for a long time been studied by hydraulic engineers (see [7,19,20] and reference cited in [4]). Mathematically, the regulation of flows in networks become an attractive theoretical basis for determining the optimal boundary controls for such networks. The networks are often modeled as single canals and junctions (or nodes) where several canals come together. Various classes of networks have been studied using the methods mentioned 
above, and several junction conditions have been proposed. We consider a subcritical flow and use junction conditions as those given in $[12,19,21,22]$. These conditions are the total flux and the energy continuity at the junction. Further detailed discussion of other junction conditions can be found in $[13,14,16,23]$.

Important results on network flows have been shown by many researchers. For example, Halleux et al. [14] have used the Riemann approach to deduce a stabilization control, for a cascade network made up by several interconnected canals. The control actions are provided by sluice gates opening at the end of each canal. The settings of these gates determine the fluid velocity at the ends of the canal. Bastin et al. [17] have used the Lyapunov stability approach on a cascade network with a bottom slop. Leugering et al. [12] have studied stabilization and null controlability of perturbations around a steady state for a star configuration network. Such a network can also be found in [11] and [21], where Li have studied the exact boundary controlability of unsteady flows. Tree configuration networks have been considered in [13,22,23].

The purpose of this paper is to investigate a class of boundary feedback controllers for star-like networks. The flow at the boundaries are the controllable quantities. The goal is to construct feedback controllers that result in the water flow and the height approaching a prescribed steady state as time goes to infinity. We apply the approach to a network of three canals, but it can be generalized to more complicated star-like networks.

The feedback boundary controllers depend on the state of the canal at earlier times. In other words, earlier data of the canal are used to build current controllers. In the practical point of view, the existence of a time delay is necessary, since there is always a time delay, between the on-line measurement data and their use to determine the controllers. Time delayed boundary feedback controllers have been derived in $[11,24]$ to stabilize the gas flow network problem around a given stationary state. Note that the approach used in [11] is not the same to our approach. The difference is in how the Lyapunov function is defined and how the rate of change of the Lyapunov function is treated to derive the delayed feedback controllers. We consider a similar network but the junction conditions are different.

Our approach consists of expressing the rate of change of energy of the linearized problem, as a second order polynomial in terms of the flow at the boundaries (free endpoints of the canals). The second order polynomial is negative for a set of controls and the stabilization rate is bounded. The boundedness is in agreement with the fact that the rate at which solutions of hyperbolic systems are affected by boundary conditions is bounded. To build the control law, the water level at the boundaries is used. The fact that the flow is stabilized for controls in an interval gives robustness and flexibility. This is essential for two reasons. To begin with, the control will be applied to the real problem, which is nonlinear. Secondly, the water level at the boundaries influences the control. These will be known by measurements, which will only be approximately correct.

The paper is organized as follows. In Sect. 2, the network, the equations and the node conditions under subcritical assumption, are presented. We also discuss how to determine a steady state solution. In Sect. 3, one derives the linearized system and corresponding characteristic variables, on which controllers are built. The main result is also formulated, stating controllers and corresponding energy decay rates. In Sect. 4 , the approach is presented to a single canal, while the case of the network is proven in Sect. 5. Numerical results obtained by a high order finite volume method $[25,26]$ are presented in Sect. 6. We have applied the approach to the real model which is nonlinear. The numerical results show the behaviour of the water level and flow for different choices of controllers. It also show the robustness of the method since the controllers work for even the nonlinear model with big perturbations.

\section{Governing equations and steady state solution}

Consider the star network canal given by Fig. 1. It is made up by three canals and one junction (denoted by $M$ ) where all canals come together. The network model is given by the 1D Saint-Venant equations in each canal, together with junction conditions at $M$. The variables in a particular canal are indexed by the canal number. For instance, for the $i$ th canal ( $i=1,2,3): h_{i}$ is the height of the fluid column $(m), q_{i}$ is the flow $\left(m^{3} s^{-1}\right), L_{i}$ and $l_{i}$ are the length and the width of the canal $(m)$, respectively. The one dimensional Saint-Venant equations considered in the present paper are the following:

$$
\begin{cases}\frac{\partial h_{i}}{\partial t}+\frac{1}{l_{i}} \frac{\partial q_{i}}{\partial x}=0, & \text { in }\left[0, L_{i}\right], \\ \frac{\partial q_{i}}{\partial t}+g l_{i} h_{i} \frac{\partial h_{i}}{\partial x}+\frac{1}{l_{i}} \frac{\partial}{\partial x}\left(\frac{q_{i}^{2}}{h_{i}}\right)=0, & \text { in }\left[0, L_{i}\right]\end{cases}
$$

together with initial condition

$h_{i}(0, x)=h_{i}^{0}(x), \quad q_{i}(0, x)=q_{i}^{0}(x)$,

and boundary conditions at the free endpoints of the network,

$$
\begin{aligned}
& q_{1}(t, 0)=q_{1,0}(t), \quad q_{2}\left(t, L_{2}\right)=q_{2, L_{2}}(t), \\
& q_{3}\left(t, L_{3}\right)=q_{3, L_{3}}(t) .
\end{aligned}
$$

The network is modeled by (1-3) and node conditions at the junction $M$. The flow is assumed to be subcritical in each canal, with the direction given by Fig. 1. For $i \in\{1,2,3\}$ we set $x_{i}$ to be the endpoint of a canal which meets the other canals at the node $M$ (i.e $x_{1}=L_{1}, x_{2}=0, x_{3}=0$ ). We also set $\epsilon_{i}=1$, if $x_{i}=L_{i}$ and $\epsilon_{i}=-1$, if $x_{i}=0$ (i.e $\left.\epsilon_{1}=1, \epsilon_{2}=-1, \epsilon_{3}=-1\right)$. 
Fig. 1 The star like network

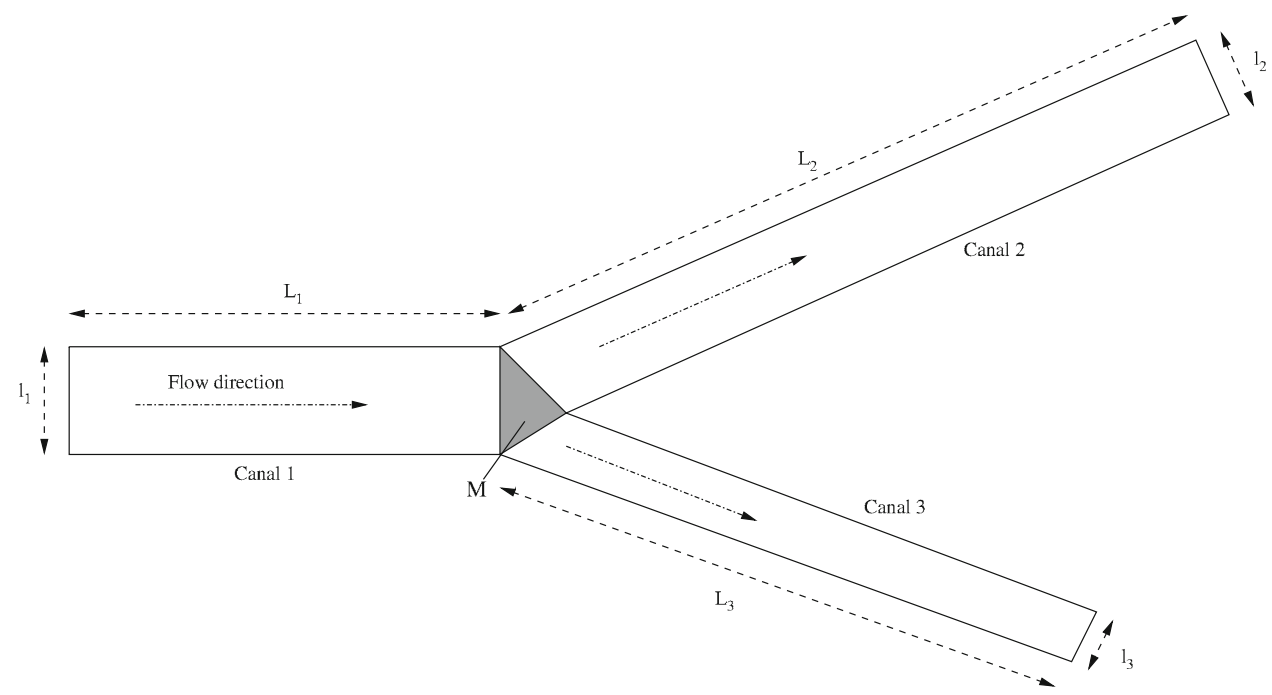

At the junction node $M$, we have the total flux and the energy continuity conditions ( $\mathrm{cf}[12,19,21,22])$. These conditions respectively are

$\sum_{i=1}^{3} \epsilon_{i} q_{i}\left(t, x_{i}\right)=0$,

$\mathcal{S}_{1}=\mathcal{S}_{j}, j=2,3$,

where

$\mathcal{S}_{i}=g h_{i}\left(t, x_{i}\right)+\frac{1}{2} \frac{q_{i}^{2}\left(t, x_{i}\right)}{l_{i}^{2} h_{i}^{2}\left(t, x_{i}\right)}$.

The controllers will be applied to the above nonlinear model. These controllers are derived from a linear system which is obtained from the linearization of the nonlinear model, using small perturbation assumptions. The perturbations are the difference between the state of the nonlinear model $\left(h_{i}, q_{i}\right)$ and a desired steady state $\left(\bar{h}_{i}, \bar{q}_{i}\right)$.

\subsection{Steady state}

We aim to achieve a prescribed steady state when time goes to infinity. The steady state solution $\left(\bar{h}_{i}, \bar{q}_{i}\right)$ is derived from (1) and (4-5) and thus satisfies the following system:

$$
\left\{\begin{array}{l}
\frac{\partial \bar{q}_{i}}{\partial x}=0, \quad \frac{\partial \bar{h}_{i}}{\partial x}=0, \quad \text { in }\left[0, L_{i}\right], \quad i=1,2,3, \\
\sum_{i=1}^{3} \epsilon_{i} \bar{q}_{i}\left(x_{i}\right)=0, \quad \text { at } M, \\
\overline{\mathcal{S}}_{1}=\overline{\mathcal{S}}_{j}, \quad j=2,3, \quad \overline{\mathcal{S}}_{i}=g \bar{h}_{i}\left(x_{i}\right)+\frac{1}{2} \frac{\bar{q}_{i}^{2}\left(x_{i}\right)}{l_{i}^{2} \bar{h}_{i}^{2}\left(x_{i}\right)} .
\end{array}\right.
$$

The flow direction (see Fig. 1) and the subcritical flow condition yield that

$\bar{q}_{i} \geq 0$ and $\sqrt{g \bar{h}_{i}}>\frac{\bar{q}_{i}}{l_{i} \bar{h}_{i}}, \quad i=1,2,3$,

respectively.

\section{Linearized model and main result}

\subsection{Linearized model}

We introduce the residual state $\left(\hat{h}_{i}, \hat{q}_{i}\right)$ as the difference between the present state $\left(h_{i}, q_{i}\right)$ and the steady state $\left(\bar{h}_{i}, \bar{q}_{i}\right): \hat{h}_{i}(t, x)=h_{i}(t, x)-\bar{h}_{i}(x), \quad \hat{q}_{i}(t, x)=q_{i}(t, x)-$ $\bar{q}_{i}(x)$. We use the assumptions $\left|\hat{h}_{i}\right| \ll \bar{h}_{i}$ and $\left|\hat{q}_{i}\right| \ll\left|\bar{q}_{i}\right|$ to linearize $(1-5)$. Therefore the solution $\left(\hat{h}_{i}, \hat{q}_{i}\right)$ satisfies

$$
\left\{\begin{array}{l}
\text { (a) } \frac{\partial \hat{h}_{i}}{\partial t}+\frac{1}{l_{i}} \frac{\partial \hat{q}_{i}}{\partial x}=0, \quad i=1,2,3, \\
\text { (b) } \frac{\partial \hat{q}_{i}}{\partial t}+\sigma_{i} \frac{\partial \hat{q}^{i}}{\partial x}+\rho_{i} \frac{\partial \hat{h}_{i}}{\partial x}=0, \quad i=1,2,3, \\
\text { (c) } \sum_{i=1}^{3} \epsilon_{i} \hat{q}_{i}\left(t, x_{i}\right)=0, \quad \text { at } M, \\
\text { (d) } \hat{\mathcal{S}}_{1}=\hat{\mathcal{S}}_{j}, \quad j=2,3, \quad \hat{\mathcal{S}}_{i}=\gamma_{i} \hat{h}_{i}\left(t, x_{i}\right)+\beta_{i} \hat{q}_{i}\left(t, x_{i}\right),
\end{array}\right.
$$

with boundary conditions

$$
\begin{aligned}
& \hat{q}_{1}(t, 0)=\hat{q}_{1,0}(t), \quad \hat{q}_{2}\left(t, L_{2}\right)=\hat{q}_{2, L_{2}}(t), \\
& \hat{q}_{3}\left(t, L_{3}\right)=\hat{q}_{3, L_{3}}(t),
\end{aligned}
$$

and initial conditions

$\hat{h}_{i}(0, x)=\hat{h}_{i}^{0}(x), \quad \hat{q}_{i}(0, x)=\hat{q}_{i}^{0}(x)$.

Here,

$$
\begin{aligned}
\sigma_{i} & =2 \frac{\bar{q}_{i}}{l_{i} \bar{h}_{i}}, \quad \rho_{i}=g l_{i} \bar{h}_{i}-\frac{\bar{q}_{i}^{2}}{l_{i} \bar{h}_{i}^{2}}, \quad \beta_{i}=\frac{\bar{q}_{i}}{l_{i}^{2} \bar{h}_{i}^{2}}=\frac{1}{2} \delta_{i} \sigma_{i}, \\
\gamma_{i} & =g-\frac{\bar{q}_{i}^{2}}{l_{i}^{2} \bar{h}_{i}^{3}}=\delta_{i} \rho_{i}, \quad \delta_{i}=\frac{1}{l_{i} \bar{h}_{i}} .
\end{aligned}
$$

Using (8), we have $\sigma_{i} \geq 0, \rho_{i}>0, \beta_{i} \geq 0$ and $\gamma_{i}>0$.

The functions $\hat{q}_{1,0}(t), \hat{q}_{2, L_{2}}(t)$ and $\hat{q}_{3, L_{3}}(t)$ are time-delay feedback control laws to be prescribed in such a way they yield exponential convergence of $\left(\hat{h}_{i}, \hat{q}_{i}\right)$ to zero. 
The system $(9 a-b)$ can be written as

$\frac{\partial U_{i}}{\partial t}+A_{i} \frac{\partial U_{i}}{\partial x}=0$

where

$U_{i}=\left(\begin{array}{c}\hat{h}_{i} \\ \hat{q}_{i}\end{array}\right)$ and $A_{i}=\left(\begin{array}{cc}0 & 1 / l_{i} \\ \rho_{i} & \sigma_{i}\end{array}\right)$.

Using the following characteristic variables

$w_{i 1}=l_{i} \hat{h}_{i}-\frac{1}{\lambda_{i 2}} \hat{q}_{i}$ and $w_{i 2}=l_{i} \hat{h}_{i}-\frac{1}{\lambda_{i 1}} \hat{q}_{i}$,

(9a-b) is now written as two independent equations as follows

$\frac{d w_{i j}}{d t}=\frac{\partial w_{i j}}{\partial t}+\lambda_{i j} \frac{\partial w_{i j}}{\partial x}=0, \quad j=1,2$.

Here, $\lambda_{i 1}$ and $\lambda_{i 2}$ are the eigenvalues of $A_{i}$ :

$\lambda_{i 1}=\frac{\bar{q}_{i}}{l_{i} \bar{h}_{i}}-c_{i} \quad$ and $\quad \lambda_{i 2}=\frac{\bar{q}_{i}}{l_{i} \bar{h}_{i}}+c_{i}, \quad c_{i}=\sqrt{g \bar{h}_{i}}$.

One can notice a relationship between $\left(\sigma_{i}, \rho_{i}\right)$ defined in (12) and $\left(\lambda_{i 1}, \lambda_{i 2}\right)$ :

$\sigma_{i}=\lambda_{i 1}+\lambda_{i 2}=\lambda_{i 2}-\left|\lambda_{i 1}\right|$ and $\rho_{i}=l_{i}\left|\lambda_{i 1}\right| \lambda_{i 2}$.

Furthermore, (8) gives

$\lambda_{i 1}<0<\lambda_{i 2}$ and $\lambda_{i 1}+\lambda_{i 2} \geq 0$,

respectively.

We rewrite $(9 \mathrm{c}-\mathrm{e})$ by expressing incoming characteristic variables at $M$ (i.e $w_{11}\left(t, L_{1}\right), w_{22}(t, 0)$ and $\left.w_{32}(t, 0)\right)$ in terms of outgoing characteristic variables at $M$ (i.e $w_{12}\left(t, L_{1}\right), w_{21}(t, 0)$ and $\left.w_{31}(t, 0)\right)$. One gets

$B\left(\begin{array}{c}w_{11}\left(t, L_{1}\right) \\ w_{22}(t, 0) \\ w_{32}(t, 0)\end{array}\right)=C\left(\begin{array}{c}w_{12}\left(t, L_{1}\right) \\ w_{21}(t, 0) \\ w_{31}(t, 0)\end{array}\right)$,

where

$B=\left(\begin{array}{ccc}\frac{\lambda_{11} \lambda_{12}}{\lambda_{12}-\lambda_{11}} & \frac{\lambda_{21} \lambda_{22}}{\lambda_{22}-\lambda_{21}} & \frac{\lambda_{31} \lambda_{32}}{\lambda_{32}-\lambda_{31}} \\ \frac{\lambda_{12}}{\lambda_{12}-\lambda_{11}} \chi_{1} & \frac{\lambda_{21}}{\lambda_{22}-\lambda_{21}} \kappa_{2} & 0 \\ \frac{\lambda_{12}}{\lambda_{12}-\lambda_{11}} \chi_{1} & 0 & \frac{\lambda_{31}}{\lambda_{32}-\lambda_{31}} \kappa_{3}\end{array}\right)$

and

$C=\left(\begin{array}{ccc}\frac{\lambda_{11} \lambda_{12}}{\lambda_{12}-\lambda_{11}} & \frac{\lambda_{21} \lambda_{22}}{\lambda_{22}-\lambda_{21}} & \frac{\lambda_{31} \lambda_{32}}{\lambda_{32}-\lambda_{31}} \\ \frac{\lambda_{11}}{\lambda_{12}-\lambda_{11}} \kappa_{1} & \frac{\lambda_{22}}{\lambda_{22}-\lambda_{21}} \chi_{2} & 0 \\ \frac{\lambda_{12}}{\lambda_{12}-\lambda_{11}} \kappa_{1} & 0 & \frac{\lambda_{32}}{\lambda_{32}-\lambda_{31}} \chi_{3}\end{array}\right)$.

Here

$\chi_{i}=\frac{\gamma_{i}}{l_{i}}+\lambda_{i 1} \beta_{i} \quad$ and $\quad \kappa_{i}=\frac{\gamma_{i}}{l_{i}}+\lambda_{i 2} \beta_{i}$.
By considering the determinants of the matrices $\mathrm{B}$ and $\mathrm{C}$ it follows straightforwardly that $B$ and $C$ are invertible if condition (16) is satisfied.

\subsection{Main result}

The feedback control of this paper relies on the fact that it is possible to express outgoing characteristic variables at the free endpoints of the network $\left(w_{11}(t, 0), w_{22}\left(t, L_{2}\right)\right.$, $\left.w_{32}\left(t, L_{3}\right)\right)$ in terms of initial data and the solution at the endpoints at earlier times. Thus, we formulate the following

Lemma 1 The outgoing characteristic variables at the free endpoints of the network, satisfy

$\left(\begin{array}{c}w_{11}(t, 0) \\ w_{22}\left(t, L_{2}\right) \\ w_{32}\left(t, L_{3}\right)\end{array}\right)=\left(\begin{array}{l}b_{1}(t) \\ b_{2}(t) \\ b_{3}(t)\end{array}\right)$

where $b_{i}, i=1,2,3$, depend only on the initial condition and the solution at the endpoints at earlier times $\tau=t-\delta t$ with $\delta t \geq \min \left(\frac{L_{1}}{\left|\lambda_{11}\right|}, \frac{L_{1}}{\lambda_{22}}, \frac{L_{3}}{\lambda_{32}}\right)$.

The proof of Lemma 1 is given in Sect. 5 .

Let us consider three arbitrary functions $\theta_{1}, \theta_{2}, \theta_{3}$ : $\left.\left.\mathbb{R}^{+} \longrightarrow\right] 0,1\right]$. We choose $\hat{q}_{1,0}, \hat{q}_{2, L_{2}}$ and $\hat{q}_{3, L_{3}}$ as follows

$\hat{q}_{1,0}(t)=\frac{\gamma_{1}}{2 l_{1} a_{1}}\left(\sqrt{1-\theta_{1}(t)}-1\right) b_{1}(t)$,

$\hat{q}_{2, L_{2}}(t)=-\frac{\gamma_{2}}{2 l_{2} a_{2}}\left(\sqrt{1-\theta_{2}(t)}-1\right) b_{2}(t)$,

$\hat{q}_{3, L_{3}}(t)=-\frac{\gamma_{3}}{2 l_{3} a_{3}}\left(\sqrt{1-\theta_{3}(t)}-1\right) b_{3}(t)$,

where $a_{i}=\frac{\delta_{i}}{2}\left(\lambda_{i 2}-\lambda_{i 1}\right)>0, \gamma_{i}$ are given by (12) and $b_{i}, i=1,2,3$, are mentioned in Lemma 1 .

In the sequel, the following notations are used:

$T_{0}=\max \left(\frac{L_{1}}{\left|\lambda_{11}\right|}, \frac{L_{2}}{\lambda_{22}}, \frac{L_{3}}{\lambda_{32}}\right)+\max \left(\frac{L_{1}}{\lambda_{12}}, \frac{L_{2}}{\left|\lambda_{21}\right|}, \frac{L_{3}}{\left|\lambda_{31}\right|}\right)$,

$t_{k}=k T_{0}, k \in \mathbb{N}, \quad Q_{i}=\left[t_{k}, t_{k+1}\right] \times\left[0, L_{i}\right]$,

$d i v \equiv\left(\frac{\partial}{\partial t}, \frac{\partial}{\partial x}\right)$

$H\left(\operatorname{div}, Q_{i}\right)=\left\{\mathcal{V} \in L^{2}\left(Q_{i}\right)^{2} ; \operatorname{div} \mathcal{V} \in L^{2}\left(Q_{i}\right)\right\}$

We define the energy of the flow in the network by

$E(t)=\sum_{i=1}^{3} \int_{0}^{L_{i}}\left(l_{i} \gamma_{i} \hat{h}_{i}^{2}+\delta_{i} \hat{q}_{i}^{2}\right) d x$

where $\gamma_{i}$ and $\delta_{i}$ are defined in (12). The main result of the paper is the following Theorem, proved in Sect. 5.4.

Theorem 1 Assume that (16) holds, the initial conditions belongs to $L^{2}\left(\left[0, L_{i}\right]\right)$ and $\left(\hat{q}_{1,0}(t), \hat{q}_{2, L_{2}}(t), \hat{q}_{3, L_{3}}(t)\right)$ satisfy 
(21). Then (9) has a solution $\left(\hat{h}_{i}, \hat{q}_{i}\right)$ satisfying the following regularity:

$$
\left(\begin{array}{c}
\hat{h}_{i} \\
\hat{q}_{i}
\end{array}\right), \quad\left(\begin{array}{c}
\hat{q}_{i} \\
\sigma_{i} \hat{q}_{i}+\rho_{i} \hat{h}_{i}
\end{array}\right) \in H\left(\operatorname{div}, Q_{i}\right) .
$$

Moreover, the energy is non-increasing in $\left[t_{k}, t_{k+1}\right]$ and satisfies

$$
E\left(t_{k+1}\right) \leq\left(1-\Theta^{k}\right) E\left(t_{k}\right) .
$$

Here, $t_{k}$ and $H\left(\right.$ div,$\left.Q_{i}\right)$ are given by (22), $E$ is given by (23), $\left.\Theta^{k}=\min \left(\Delta_{1}^{k}, \Delta_{2}^{k}, \Delta_{3}^{k}\right) \in\right] 0,1[$,

$\Delta_{1}^{k}=\min \left(\frac{\left|\lambda_{11}\right|}{\lambda_{12}+\left|\lambda_{11}\right|} \inf _{x \in\left[0, L_{1}\right]} \theta_{1}\left(t_{k}+\frac{x}{\left|\lambda_{11}\right|}\right), \frac{\gamma_{1} \epsilon}{l_{1}}\right)$,

$\Delta_{2}^{k}=\min \left(\frac{\left|\lambda_{21}\right|}{\lambda_{22}+\left|\lambda_{21}\right|} \inf _{x \in\left[0, L_{2}\right]} \theta_{2}\left(t_{k}+\frac{L_{2}-x}{\lambda_{22}}\right), \frac{\gamma_{2} \epsilon}{l_{2}}\right)$,

$\Delta_{3}^{k}=\min \left(\frac{\left|\lambda_{31}\right|}{\lambda_{32}+\left|\lambda_{31}\right|} \inf _{x \in\left[0, L_{3}\right]} \theta_{3}\left(t_{k}+\frac{L_{3}-x}{\lambda_{32}}\right), \frac{\gamma_{3} \epsilon}{l_{3}}\right)$,

and $\epsilon>0$ is given by equivalence of norms (see the Appendix).

Remark 1 1. Owing to Lemma 1, the controllers (21) are built on the solution at earlier times. Since $\gamma_{i}$ and $a_{i}$ are constant, then due to (25), the controllers tend to zero when time goes to infinity.

2. Denoting $v^{k}=-\ln \left(\left(1-\Theta^{k}\right)^{\frac{1}{t_{1}}}\right)$, one has

$$
E\left(t_{k+1}\right) \leq E\left(t_{k}\right) \exp \left(-v^{k} t_{1}\right) .
$$

Therefore, defining $\mu^{k}=\frac{1}{k} \sum_{j=0}^{k-1} v^{j}$, we obtain

$$
E\left(t_{k}\right) \leq E(0) \exp \left(-\mu^{k} t_{k}\right)
$$

Thus, the functions $\theta$ can be viewed as a stabilization rate for the exponential decrease. The closer they are to 1 , the faster the exponential decrease is.

\section{Building the controllers for a single canal}

We construct a stabilization process for a single canal, which should drive the perturbations $\hat{h}$ and $\hat{q}$ to zero exponentially in time. We consider the Saint-Venant equations (1) without the index $i$ standing for the canal number :

$$
\begin{cases}\frac{\partial h}{\partial t}+\frac{1}{l} \frac{\partial q}{\partial x}=0, & \text { in }[0, L], \\ \frac{\partial q}{\partial t}+g \operatorname{lh} \frac{\partial h}{\partial x}+\frac{1}{l} \frac{\partial}{\partial x}\left(\frac{q^{2}}{h}\right)=0, & \text { in }[0, L],\end{cases}
$$

together with initial condition

$h(0, x)=h^{0}(x), \quad q(0, x)=q^{0}(x)$, and boundary conditions

$q(t, 0)=q_{0}(t), \quad q(t, L)=q_{L}(t)$.

The steady state solution satisfies

$\frac{\partial \bar{q}}{\partial x}=0, \quad \frac{\partial \bar{h}}{\partial x}=0$,

and the linearized model is

$\left\{\begin{array}{l}\text { (a) } \frac{\partial \hat{h}}{\partial t}+\frac{1}{l} \frac{\partial \hat{q}}{\partial x}=0, \\ \text { (b) } \frac{\partial \hat{q}}{\partial t}+\sigma \frac{\partial \hat{q}}{\partial x}+\rho \frac{\partial \hat{h}}{\partial x}=0,\end{array}\right.$

together with the initial condition

$\hat{h}(0, x)=\hat{h}^{0}(x), \quad \hat{q}(0, x)=\hat{q}^{0}(x)$,

and the boundary conditions as time-delay feedback control law

$\hat{q}(t, 0)=\hat{q}_{0}(t), \quad \hat{q}(t, L)=\hat{q}_{L}(t)$.

Here,

$\sigma=2 \frac{\bar{q}}{l \bar{h}}, \quad \rho=g l \bar{h}-\frac{\bar{q}^{2}}{l \bar{h}^{2}}$.

The characteristic variables are

$w_{1}=l \hat{h}-\frac{1}{\lambda_{2}} \hat{q} \quad$ and $\quad w_{2}=l \hat{h}-\frac{1}{\lambda_{1}} \hat{q}$

where $\lambda_{1}=\frac{\bar{q}}{l \bar{h}}-\sqrt{g \bar{h}}$ and $\lambda_{2}=\frac{\bar{q}}{l \bar{h}}+\sqrt{g \bar{h}}$. The characteristic variables satisfy

$\frac{d w_{j}}{d t}=\frac{\partial w_{j}}{\partial t}+\lambda_{j} \frac{\partial w_{j}}{\partial x}=0, \quad j=1,2$.

Due to the subcritical flow condition and the flow direction, we have

$\lambda_{1}<0<\lambda_{2}$ and $\lambda_{1}+\lambda_{2} \geq 0$.

Furthermore $\sigma=\lambda_{1}+\lambda_{2}=\lambda_{2}-\left|\lambda_{1}\right|$ and $\rho=l\left|\lambda_{1}\right| \lambda_{2}$.

4.1 A priori energy estimation

Let $E$ be the energy of (30) defined as

$E(t)=\int_{0}^{L}\left(l \rho \hat{h}^{2}(t)+\hat{q}^{2}(t)\right) d x$,

where $\rho$ is defined in (33).

We consider the following system as a weak formulation of (30) 


$$
\left\{\begin{array}{l}
\forall(\psi, \phi) \in H^{1}(] 0, L[), \\
\int_{0}^{L} l \rho \psi \frac{\partial \hat{h}}{\partial t} d x-\int_{0}^{L} \hat{q} \frac{\partial(\psi \rho)}{\partial x} d x \\
\quad+\rho \psi(L) \hat{q}_{L}(t)-\rho \psi(0) \hat{q}_{0}(t)=0, \\
\int_{0}^{L} \phi \frac{\partial \hat{q}}{\partial t} d x-\int_{0}^{L} \hat{q} \frac{\partial(\sigma \phi)}{\partial x} d x+\int_{0}^{L} \phi \frac{\partial(\rho \hat{h})}{\partial x} d x \\
+\sigma \phi(L) \hat{q}_{L}(t)-\sigma \phi(0) \hat{q}_{0}(t)=0,
\end{array}\right.
$$

together with boundary and initial conditions.

We give the above weak formulation because we will prove the existence of weak solution for the single canal (Theorem 3). A corresponding result for the network is Theorem 1.

Let us estimate the variation of the energy $E$ in the canal in order to define the controllers $\hat{q}_{L}(t)$ at $\{x=L\}$ and $\hat{q}_{0}(t)$ at $\{x=0\}$. For this purpose we let $(\psi, \phi)=(\hat{h}, \hat{q})$ in $(38)$ to get

$$
\begin{aligned}
\frac{1}{2} \frac{d}{d t} E(t)= & -\frac{1}{2} \sigma \hat{q}_{L}^{2}(t)-\rho \hat{h}(t, L) \hat{q}_{L}(t) \\
& +\frac{1}{2} \sigma \hat{q}_{0}^{2}(t)+\rho \hat{h}(t, 0) \hat{q}_{0}(t) .
\end{aligned}
$$

One difference between our control method and many other, is the way the energy is defined and how the time variation of the energy is treated $[14,17,27]$. In [17,27], the energy is defined by weighted Lyapunov function and a stability analysis is used for the RHS of the time variation of the energy. In our case, a relationship between $\hat{h}$ and $\hat{q}$ is used to express the RHS of (39) as a second order polynomial in terms of $\hat{q}$. That second order polynomial is treated in such a way to obtain an exponential decrease of the energy.

\subsection{Time-delay boundary feedback design for a single canal and the stabilization process}

In this section, we assume smooth initial data and boundary conditions. The results for $L^{2}$ data are given in Sect. 4.3. To build the feedback control laws, we express the height at the boundary in terms of the flow and outgoing characteristic variables. By tracing characteristics backward in time, as indicated in Fig. 2 and using (14), we express outgoing characteristic variables:

$$
\left(\begin{array}{l}
w_{1}\left(\tau_{2}, 0\right) \\
w_{2}\left(\tau_{1}, L\right)
\end{array}\right)=\left(\begin{array}{l}
c_{1}\left(\tau_{2}\right) \\
c_{2}\left(\tau_{1}\right)
\end{array}\right)
$$

where

$c_{1}\left(\tau_{2}\right)=\left\{\begin{array}{l}\left.\left.w_{1}\left(0, x_{0}\right), \quad x_{0}=\left|\lambda_{1}\right| \tau_{2}, \quad \tau_{2} \in\right] 0, L /\left|\lambda_{1}\right|\right], \\ w_{1}\left(\tau_{2}-L /\left|\lambda_{1}\right|, L\right), \quad \tau_{2} \geq L /\left|\lambda_{1}\right|,\end{array}\right.$

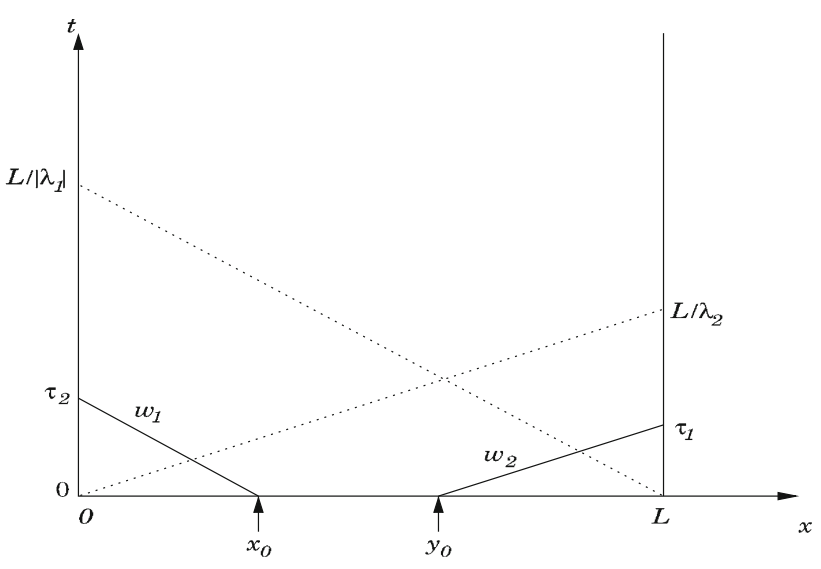

(a) The starting points $\left(x_{0}\right.$ and $\left.y_{0}\right)$ of the characteristics are within the canal. This occurs when $\tau_{2} \leq L /\left|\lambda_{1}\right|$ and $\tau_{1} \leq L / \lambda_{2}$. Thus, the initial condition is concerned, since these points correspond to the time $t=0$.

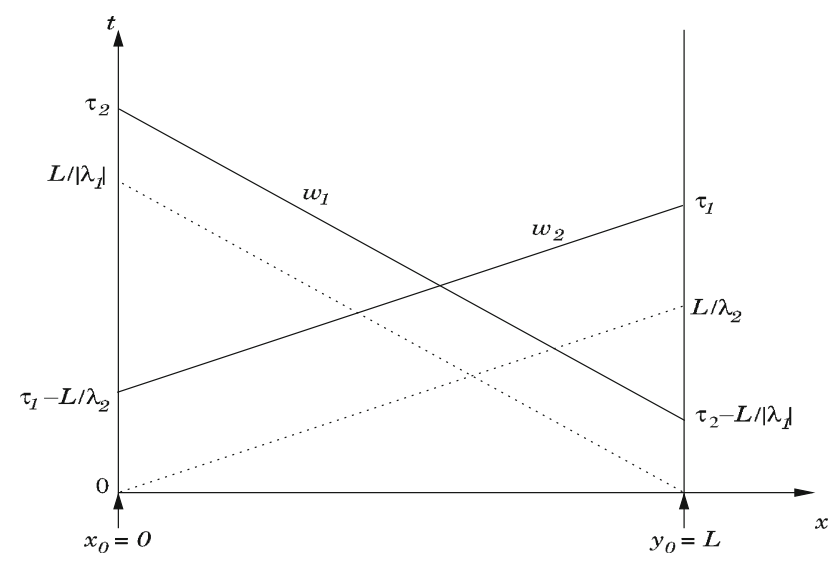

(b) The starting points $\left(x_{0}\right.$ and $\left.y_{0}\right)$ of the characteristics are located at the endpoints of the canal. This occurs when $\tau_{2} \geq L /\left|\lambda_{1}\right|$ and $\tau_{1} \geq L / \lambda_{2}$. Thus, the canal status at the endpoints at times $\tau_{2}-L /\left|\lambda_{1}\right|$ and $\tau_{1}-$ $L / \lambda_{2}$ is concerned.

Fig. 2 Outgoing characteristics at the endpoints of the canal and their starting points

$c_{2}\left(\tau_{1}\right)=\left\{\begin{array}{l}\left.\left.w_{2}\left(0, y_{0}\right), y_{0}=L-\lambda_{2} \tau_{1}, \tau_{1} \in\right] 0, L / \lambda_{2}\right], \\ w_{2}\left(\tau_{1}-L / \lambda_{2}, 0\right), \quad \tau_{1} \geq L / \lambda_{2} .\end{array}\right.$

From (35), one has

$\hat{h}\left(\tau_{1}, L\right)=\frac{1}{l \lambda_{1}} \hat{q}_{L}\left(\tau_{1}\right)+\frac{1}{l} w_{2}\left(\tau_{1}, L\right)$,
$\hat{h}\left(\tau_{2}, 0\right)=\frac{1}{l \lambda_{2}} \hat{q}_{0}\left(\tau_{2}\right)+\frac{1}{l} w_{1}\left(\tau_{2}, 0\right)$.

Considering the energy equation (39), one deduces from (41-44) that 


$$
\begin{aligned}
\frac{1}{2} \frac{d E}{d t}(t)= & a \hat{q}_{0}^{2}(t)+\frac{\rho}{l} c_{1}(t) \hat{q}_{0}(t) \\
& +a \hat{q}_{L}^{2}(t)-\frac{\rho}{l} c_{2}(t) \hat{q}_{L}(t),
\end{aligned}
$$

where $a=\frac{1}{2}\left(\lambda_{2}+\left|\lambda_{1}\right|\right), \rho$ is defined in (33) and $c_{1}(t)$ and $c_{2}(t)$ are given by (41) and (42), respectively.

The idea is to prescribe the flow at the endpoints so that we get an exponential decay of energy. To this end, let us formulate an observation for second order polynomials as a Lemma.

Lemma 2 Consider a second order polynomial $P(q)=$ $a q^{2}+b q$, where $a>0$. For any $\left.\left.\theta \in\right] 0,1\right]$,

$P\left(\frac{b}{2 a}(\sqrt{1-\theta}-1)\right)=-\frac{b^{2}}{4 a} \theta$.

In our control method, the flow at the boundary is prescribed as follows:

$$
\begin{aligned}
& \hat{q}_{L}(t)=\frac{\rho c_{2}(t)}{2 a l}\left(\sqrt{1-\theta_{2}(t)}-1\right) \\
& \hat{q}_{0}(t)=-\frac{\rho c_{1}(t)}{2 a l}\left(\sqrt{1-\theta_{1}(t)}-1\right),
\end{aligned}
$$

where $\left.\left.\theta_{1}, \theta_{2}: \mathbb{R}^{+} \longrightarrow\right] 0,1\right]$.

Remark 2 The controllers defined in (47) are time-delay boundary feedback controllers. In fact, the expressions of $c_{1}(t)$ and $c_{2}(t)$ defined in (41) and (42), and used in (47) involve a time-delay. The controllers at time $t$ depend on the initial condition or on the solution at earlier time $t-\delta t$, where $\delta t=L /\left|\lambda_{1}\right|$ for the upstream controller and $\delta t=L / \lambda_{2}$ for the downstream controller. From a practical point of view, there is always a delay between the on-line measurements of data and their to build the controllers. This is not the case in [12, 14, 15, 17].

On the other hand, the only on-line measurements of the water levels at the endpoints, is sufficient to determine the controllers, as in [14,15] and [17]. This is also true for the network.

With the controllers given by (47), it follows by Lemma 2, that (45) becomes

$\frac{1}{2} \frac{d E}{d t}(t)=-\frac{\rho^{2} c_{1}^{2}(t)}{4 a l^{2}} \theta_{1}(t)-\frac{\rho^{2} c_{2}^{2}}{4 a l^{2}} \theta_{2}(t)$.

Thus, we get the following

Theorem 2 Let $t_{k}=k L /\left|\lambda_{1}\right|, k \in \mathbb{N}$. Assume that (36) holds, the initial condition $\left(\hat{h}^{0}, \hat{q}^{0}\right)$ is continuous in $[0, L]$. Also, that $\theta_{1}$ and $\theta_{2}$ are continuous in $\left.] 0,1\right]$, and $\hat{q}_{0}$ and $\hat{q}_{L}$ satisfy (47). Then (30) has a unique solution $(\hat{h}, \hat{q})$ continuous in $\left[t_{k}, t_{k+1}\right] \times[0, L]$ such that the energy is nonincreasing in $\left[t_{k}, t_{k+1}\right]$ and satisfies:

$E\left(t_{k+1}\right) \leq\left(1-\Theta^{k}\right) E\left(t_{k}\right)$, where $E$ is given by (37) and $\left.\Theta^{k}=\frac{\left|\lambda_{1}\right|}{\lambda_{2}+\left|\lambda_{1}\right|} \Xi_{k} \in\right] 0,1[$

$\Xi_{k}=\min \left(\inf _{x \in] 0, L[} \theta_{2}\left(t_{k}+\frac{L-x}{\lambda_{2}}\right), \inf _{x \in] 0, L[} \theta_{1}\left(t_{k}+\frac{x}{\left|\lambda_{1}\right|}\right)\right)$.

Proof The existence and uniticity of the solution follow by (34) and constructions (41-42).

Integrating (48) from 0 to $t_{1}$, we have

$$
\begin{aligned}
E\left(L /\left|\lambda_{1}\right|\right)= & E(0)-\frac{\rho^{2}}{l^{2}} \int_{0}^{L /\left|\lambda_{1}\right|} \frac{\left(c_{2}(t)\right)^{2}}{2 a} \theta_{2}(t) d t \\
& -\frac{\rho^{2}}{l^{2}} \int_{0}^{L /\left|\lambda_{1}\right|} \frac{\left(c_{1}(t)\right)^{2}}{2 a} \theta_{1}(t) d t \\
\leq & E(0)-\frac{\rho^{2}}{l^{2}} \int_{0}^{L / \lambda_{2}} \frac{\left(c_{2}(t)\right)^{2}}{2 a} \theta_{2}(t) d t \\
& -\frac{\rho^{2}}{l^{2}} \int_{0}^{L /\left|\lambda_{1}\right|} \frac{\left(c_{1}(t)\right)^{2}}{2 a} \theta_{1}(t) d t
\end{aligned}
$$

Using (41) and (42) we get

$$
\begin{aligned}
& E\left(L /\left|\lambda_{1}\right|\right) \leq E(0)-\frac{\rho^{2}}{l^{2}} \int_{0}^{L / \lambda_{2}} \frac{w_{2}^{2}\left(0, L-\lambda_{2} t\right) \theta_{2}(t)}{2 a} d t \\
& -\frac{\rho^{2}}{l^{2}} \int_{0}^{L /\left|\lambda_{1}\right|} \frac{w_{1}^{2}\left(0,\left|\lambda_{1}\right| t\right) \theta_{1}(t)}{2 a} d t \\
& \leq E(0)-\frac{\rho^{2}}{l^{2}} \int_{0}^{L} \frac{w_{2}^{2}(0, x) \theta_{2}\left(\frac{L-x}{\lambda_{2}}\right)}{2 a \lambda_{2}} d x \\
& -\frac{\rho^{2}}{l^{2}} \int_{0}^{L} \frac{w_{1}^{2}(0, x) \theta_{1}\left(\frac{x}{\left|\lambda_{1}\right|}\right)}{2 a\left|\lambda_{1}\right|} d x \\
& \leq E(0)-\frac{\rho^{2}}{l^{2}} \int_{0}^{L} \frac{w_{2}^{2}(0, x) \theta_{2}\left(\frac{L-x}{\lambda_{2}}\right)}{2 a \lambda_{2}} d x \\
& -\frac{\rho^{2}}{l^{2}} \int_{0}^{L} \frac{w_{1}^{2}(0, x) \theta_{1}\left(\frac{x}{\left|\lambda_{1}\right|}\right)}{2 a \lambda_{2}} d x \\
& \leq E(0)-\frac{\rho^{2}}{2 l^{2} a \lambda_{2}} \int_{0}^{L}\left[w_{2}^{2}(0, x)+w_{1}^{2}(0, x)\right] \Psi d x,
\end{aligned}
$$

where

$\Psi=\min \left(\theta_{2}\left(\frac{L-x}{\lambda_{2}}\right), \theta_{1}\left(\frac{x}{\left|\lambda_{1}\right|}\right)\right)$. 
On the other hand, one has the following estimation

$$
\begin{aligned}
w_{2}^{2}(0, x)+w_{1}^{2}(0, x)= & 2\left(l \hat{h}^{0}(x)\right)^{2}+\left(\frac{1}{\lambda_{1}^{2}}+\frac{1}{\lambda_{2}^{2}}\right)\left(\hat{q}^{0}(x)\right)^{2} \\
& -2\left(\frac{1}{\lambda_{1}}+\frac{1}{\lambda_{2}}\right) l \hat{h}^{0}(x) \hat{q}^{0}(x), \\
\geq & 2\left(l \hat{h}^{0}(x)\right)^{2}+\left(\frac{1}{\lambda_{1}^{2}}+\frac{1}{\lambda_{2}^{2}}\right)\left(\hat{q}^{0}(x)\right)^{2} \\
& -\left(l \hat{h}^{0}(x)\right)^{2}-\left(\frac{1}{\lambda_{1}}+\frac{1}{\lambda_{2}}\right)^{2}\left(\hat{q}^{0}(x)\right)^{2}, \\
\geq & \left(l \hat{h}^{0}(x)\right)^{2}-2 \frac{1}{\lambda_{1} \lambda_{2}}\left(\hat{q}^{0}(x)\right)^{2}, \\
\geq & \left(l \hat{h}^{0}(x)\right)^{2}+2 \frac{l^{2}}{l \rho}\left(\hat{q}^{0}(x)\right)^{2}, \\
\geq & \frac{l}{\rho}\left(l \rho\left(\hat{h}^{0}(x)\right)^{2}+\left(\hat{q}^{0}(x)\right)^{2}\right) .
\end{aligned}
$$

Therefore we deduce from (50-51) that

$$
\begin{aligned}
E\left(L /\left|\lambda_{1}\right|\right) & \leq E(0)-\Theta^{0} \int_{0}^{L}\left(l \rho\left(\hat{h}^{0}\right)^{2}+\left(\hat{q}^{0}\right)^{2}\right) d x \\
& \leq\left(1-\Theta^{0}\right) E(0) .
\end{aligned}
$$

where

$\Theta^{0}=\frac{\left|\lambda_{1}\right|}{\lambda_{2}+\left|\lambda_{1}\right|} \min \left(\inf _{x \in] 0, L[} \theta\left(\frac{L-x}{\lambda_{2}}\right), \inf _{x \in] 0, L[} \theta\left(\frac{x}{\left|\lambda_{1}\right|}\right)\right)$.

To generalize (52) with respect to time, we consider the initial condition at time $t_{k}=k L /\left|\lambda_{1}\right|$. We let

$c_{1}(t)=w_{1}\left(t_{k},\left|\lambda_{1}\right|\left(t-t_{k}\right)\right)$, if $\left.t \in\right] t_{k}, t_{k}+L /\left|\lambda_{1}\right|[$,

$c_{2}(t)=-w_{2}\left(t_{k}, L-\lambda_{2}\left(t-t_{k}\right)\right)$, if $\left.t \in\right] t_{k}, t_{k}+L / \lambda_{2}[$,

and

$\Theta^{k}=\frac{\left|\lambda_{1}\right|}{\lambda_{2}-\lambda_{1}} \Xi_{k}$

where

$\Xi_{k}=\min \left(\inf _{x \in] 0, L[} \theta\left(t_{k}+\frac{L-x}{\lambda_{2}}\right), \inf _{x \in] 0, L[} \theta\left(t_{k}+\frac{x}{\left|\lambda_{1}\right|}\right)\right)$.

Therefore by integrating from $t_{k}$ to $t_{k+1}$ and using the same arguments as for the interval $\left[0, t_{1}\right]$, the proof of Theorem 2 is finished.

Remark 3 Note that it is possible to drive the perturbations $\hat{h}$ and $\hat{q}$ to zero exponentially in time by controlling only with one free endpoint. For instance, let us consider the free endpoint $x=L$. In this case, we let $\hat{q}_{0}(t)=0$ and

$\hat{q}_{L}(t)=\frac{b(t)}{2 a}(\sqrt{1-\theta(t)}-1)$,

where

$b(t)=-\frac{\rho}{l}\left\{\begin{array}{l}\left.w_{2}\left(0, L-\lambda_{2} t\right), \quad \text { if } t \in\right] 0, L / \lambda_{2}[, \\ \left.w_{1}\left(0,-\lambda_{1}\left(t-L / \lambda_{2}\right)\right), \quad \text { if } t \in\right] L / \lambda_{2}, L T[ \\ w_{1}(t-L T, L), \quad \text { if } t \geq L T,\end{array}\right.$

$\theta \in[0,1]$ and $T=\frac{1}{\lambda_{2}}+\frac{1}{\left|\lambda_{1}\right|}$.

\subsection{A regularity result}

In this section, we consider initial data in $L^{2}$ space instead of smooth initial data as in Theorem 2 .

Theorem 3 Let $t_{k}=k L /\left|\lambda_{1}\right|, k \in \mathbb{N}$. Assume that (36) holds, $\left(\hat{h}^{0}, \hat{q}^{0}\right)$ belongs to $\left(L^{2}([0, L])\right)^{2}$ and $\hat{q}_{L}(t), \hat{q}_{0}(t)$ satisfy (47). Then the linear system (30) has a solution $(\hat{h}, \hat{q})$ satisfying the following regularity:

$$
\left(\begin{array}{c}
l \hat{h} \\
\hat{q}
\end{array}\right), \quad\left(\begin{array}{c}
\hat{q} \\
\sigma \hat{q}+\rho \hat{h}
\end{array}\right) \in H(\operatorname{div}, Q) .
$$

Moreover, the energy is non-increasing in $\left[t_{k}, t_{k+1}\right]$ and satisfies

$E\left(t_{k+1}\right) \leq\left(1-\Theta^{k}\right) E\left(t_{k}\right)$,

where $\Theta^{k}$ is given in Theorem 2.

Proof Since $\left(\hat{h}^{0}, \hat{q}^{0}\right) \in\left(L^{2}([0, L])\right)^{2}$, there exist subsequences $\left(\hat{h}_{n}\right)_{n \geq 0} \subset C^{0}([0, L])$ and $\left(\hat{q}_{n}\right)_{n \geq 0} \subset C^{0}([0, L])$ such that

$\hat{h}_{n}^{0} \longrightarrow \hat{h}^{0}$ and $\hat{q}_{n}^{0} \longrightarrow \hat{q}^{0}$ in $L^{2}([0, L]), \quad n \longrightarrow+\infty$.

where $C_{p}(] 0, L[)$ denotes the set of piecewise continuous functions in $] 0, L[$.

For any $n \geq 0$, we consider that $\left(\hat{h}_{n}, \hat{q}_{n}\right)$ satisfies the weak formulation (38) and define $\hat{q}_{0, n}(t)$ and $\hat{q}_{L, n}(t)$ as in (47) where $\hat{h}^{0}$ and $\hat{q}^{0}$ are replaced by $\hat{h}_{n}^{0}$ and $\hat{q}_{n}^{0}$, respectively. One gets the energy estimation (49) for $\left(\hat{h}_{n}, \hat{q}_{n}\right)$, which can be written as follows

$E^{n}\left(t_{k+1}\right) \leq \prod_{i=0}^{k}\left(1-\Theta^{k}\right) E^{n}(0)$,

where $E^{n}(t)=\left\|\sqrt{l \rho} \hat{h}_{n}(t)\right\|_{2, \Omega}^{2}+\left\|\hat{q}_{n}(t)\right\|_{2, \Omega}^{2}, \Theta^{k}$ and $t_{k}$ are defined in Theorem 2.

We have from (30) and (56) that

$\left(\begin{array}{c}l \hat{h}_{n} \\ \hat{q}_{n}\end{array}\right), \quad\left(\begin{array}{c}\hat{q}_{n} \\ \sigma \hat{q}_{n}+\rho \hat{h}_{n}\end{array}\right) \in H(\operatorname{div}, Q)$,

and bounded in $H($ div,$Q)$.

Using the fact that $L^{2}\left(0, T ; L^{2}(\Omega)^{2}\right)$ is a reflexive Banach space, for all $T>0$, and [28], one can show that there exists subsequences weakly converging to $(\hat{h}, \hat{q})$ in $L^{2}\left(0, T ; L^{2}(\Omega)^{2}\right)$. Moreover, $(\hat{h}, \hat{q})$ satisfies the linear system (30) together with the initial condition (31) and boundary conditions (32). Since $E(t) \leq \liminf _{n \rightarrow \infty} E^{n}(t),(49)$ is obtained from (56) and the proof of Theorem is completed.

\section{Building the controller for the star-like network}

Taking advantages of the previous sections concerning the controllers building, we will now show how to construct timedelay feedback controllers which stabilize the network. 


\subsection{A priori energy estimation}

We consider the energy of the flow in the network, given by (23). In order to take into account the junction conditions in the weak formulation for the network, we derive the following equations: For $\forall\left(\psi_{i}, \phi_{i}\right) \in\left(H^{1}(] 0, L_{i}[)\right)^{2}$, one gets from $(9 c)$ and $(9 d)$ that

$$
\begin{aligned}
\sum_{i=1}^{3} \epsilon_{i} \gamma_{i} \psi\left(x_{i}\right) \hat{q}_{i}\left(t, x_{i}\right)= & \sum_{i=1}^{2} \Upsilon_{i} \epsilon_{i} \hat{q}_{i}\left(t, x_{i}\right), \\
\sum_{i=1}^{3} \epsilon_{i} \beta_{i} \phi\left(x_{i}\right) \hat{q}_{i}\left(t, x_{i}\right)= & \hat{\mathcal{S}}_{1} \sum_{i=1}^{3} \epsilon_{i} \phi\left(x_{i}\right) \\
& -\sum_{i=1}^{3} \epsilon_{i} \phi\left(x_{i}\right) \gamma_{i} \hat{h}_{i}\left(t, x_{i}\right) .
\end{aligned}
$$

where $\hat{\mathcal{S}}_{1}$ is defined in (9) and $\Upsilon_{i}=\gamma_{i} \psi\left(x_{i}\right)-\gamma_{3} \psi\left(x_{3}\right)$.

Therefore we consider the following equations as a weak formulation for the network. For the mass conservation law: $\forall \psi_{i} \in H^{1}(] 0, L_{i}\left[\right.$ ), we multiply (9a) by $l_{i} \gamma_{i} \psi_{i}$, integrate by parts and sum over $i$, to get

$$
\begin{aligned}
& \sum_{i=1}^{3} \int_{0_{i}}^{L_{i}}\left[l_{i} \gamma_{i} \psi_{i} \frac{\partial \hat{h}_{i}}{\partial t}-\hat{q}_{i} \frac{\partial\left(\psi_{i} \gamma_{i}\right)}{\partial x}\right] d x+\sum_{i=1}^{2} \Upsilon_{i} \epsilon_{i} \hat{q}_{i}\left(t, x_{i}\right) \\
& -\gamma_{1} \psi(0) \hat{q}_{1,0}(t)+\gamma_{2} \psi\left(L_{2}\right) \hat{q}_{2, L_{2}}(t)+\gamma_{3} \psi\left(L_{3}\right) \hat{q}_{3, L_{3}}(t) \\
& \quad=0
\end{aligned}
$$

For the momentum conservation law: $\forall \phi_{i} \in H^{1}(] 0, L_{i}[)$, we multiply (9b) by $\delta_{i} \phi_{i}$, integrate by parts and sum over $i$, to get

$$
\begin{aligned}
& \sum_{i=1}^{3} \int_{0_{i}}^{L_{i}}\left[\delta_{i} \phi_{i} \frac{\partial \hat{q}_{i}}{\partial t}-2 \hat{q}_{i} \frac{\partial\left(\beta_{i} \phi_{i}\right)}{\partial x}-\hat{h}_{i} \frac{\partial\left(\gamma_{i} \phi_{i}\right)}{\partial x}\right] d x \\
& \quad+\sum_{i=1}^{3} \epsilon_{i} \phi\left(x_{i}\right)\left(2 \hat{\mathcal{S}}_{1}-\gamma_{i} \hat{h}_{i}\left(t, x_{i}\right)\right)-2 \beta_{1} \phi(0) \hat{q}_{1,0}(t) \\
& \quad+2 \beta_{2} \phi\left(L_{2}\right) \hat{q}_{2, L_{2}}(t)+2 \beta_{3} \phi\left(L_{3}\right) \hat{q}_{3, L_{3}}(t)-\gamma_{1} \phi(0) \hat{h}_{1}(t, 0) \\
& \quad+\gamma_{2} \phi\left(L_{2}\right) \hat{h}_{2}\left(t, L_{2}\right)+\gamma_{3} \phi\left(L_{3}\right) \hat{h}_{3}\left(t, L_{3}\right)=0 .
\end{aligned}
$$

In order to define the controllers, we let $\left(\psi_{i}, \phi_{i}\right)=\left(\hat{h}_{i}, \hat{q}_{i}\right)$ in (60-61). Then

$$
\begin{aligned}
\frac{1}{2} \frac{d}{d t} E(t)= & -\beta_{2} \hat{q}_{2, L_{2}}^{2}(t)-\gamma_{2} \hat{h}_{2}\left(t, L_{2}\right) \hat{q}_{2, L_{2}}(t) \\
& -\beta_{3} \hat{q}_{3, L_{3}}^{2}(t)-\gamma_{3} \hat{h}_{3}\left(t, L_{3}\right) \hat{q}_{3, L_{3}}(t)+\beta_{1} \hat{q}_{1,0}^{2}(t) \\
& +\gamma_{1} \hat{h}_{1}(t, 0) \hat{q}_{1,0}(t)-2 \hat{\mathcal{S}}_{1} \sum_{i=1}^{3} \epsilon_{i} \hat{q}_{i}\left(t, x_{i}\right) \\
& +\sum_{i=1}^{3} \epsilon_{i} \gamma_{i} \hat{q}_{i}\left(t, x_{i}\right) \hat{h}_{i}\left(t, x_{i}\right)-\sum_{i=1}^{2} \Upsilon_{i} \epsilon_{i} \hat{q}_{i}\left(t, x_{i}\right) .
\end{aligned}
$$

Meanwhile, we have the following

Lemma 3 If $(9 \mathrm{c}-9 \mathrm{~d})$ are satisfied then

$$
\begin{aligned}
& -2 \hat{\mathcal{S}}_{1} \sum_{i=1}^{3} \epsilon_{i} \hat{q}_{i}\left(t, x_{i}\right)+\sum_{i=1}^{3} \epsilon_{i} \gamma_{i} \hat{q}_{i}\left(t, x_{i}\right) \hat{h}_{i}\left(t, x_{i}\right) \\
& -\sum_{i=1}^{2} \Upsilon_{i} \epsilon_{i} \hat{q}_{i}\left(t, x_{i}\right)=0 .
\end{aligned}
$$

Proof If (9c) is satisfies then,

$2 \hat{\mathcal{S}}_{1} \sum_{i=1}^{3} \epsilon_{i} \hat{q}_{i}\left(t, x_{i}\right)=0$.

On the other hand, if we let $\psi_{i}=\hat{h}_{i}$ in (58) we get

$\sum_{i=1}^{2} \Upsilon_{i} \epsilon_{i} \hat{q}_{i}\left(t, x_{i}\right)=\sum_{i=1}^{3} \epsilon_{i} \gamma_{i} \hat{h}_{i}\left(t, x_{i}\right) \hat{q}_{i}\left(t, x_{i}\right)$

Therefore the proof of Lemma 3 is finished.

By Lemma (3), only terms from the free endpoints of the network remain in (62):

$$
\begin{aligned}
\frac{1}{2} \frac{d}{d t} E(t)=- & \beta_{2} \hat{q}_{2, L_{2}}^{2}(t)-\gamma_{2} \hat{h}_{2}\left(t, L_{2}\right) \hat{q}_{2, L_{2}}(t)-\beta_{3} \hat{q}_{3, L_{3}}^{2}(t) \\
& -\gamma_{3} \hat{h}_{3}\left(t, L_{3}\right) \hat{q}_{3, L_{3}}(t)+\beta_{1} \hat{q}_{1,0}^{2}(t) \\
& +\gamma_{1} \hat{h}_{1}(t, 0) \hat{q}_{1,0}(t)
\end{aligned}
$$

5.2 Time-delay boundary feedback controller design for the star network

As in Sect. 4, we use the relationship between the height and the flow in order to rewrite the RHS of (63) in terms of the flow and characteristic variables.

The points referred to, in this section are defined in Fig. 3. We give the proof of Lemma 1.

Proof of Lemma 1: We distinguish two cases:

Case A When the outgoing characteristic at the free endpoint, starts from a point inside the domain (dashed lines in Fig. 3).

This case occurs when $t \leq \frac{L_{1}}{\left|\lambda_{11}\right|}$ for canal $1, t \leq \frac{L_{2}}{\lambda_{22}}$ for canal 2 and $t \leq \frac{L_{3}}{\lambda_{32}}$ for canal 3. Using (14), the following relations hold

$w_{11}(t, 0)=w_{11}\left(0, y_{1}\right), \quad y_{1}=\left|\lambda_{11}\right| t$,

$w_{22}\left(t, L_{2}\right)=w_{22}\left(0, y_{2}\right), \quad y_{2}=L_{2}-\lambda_{22} t$,

$w_{32}\left(t, L_{3}\right)=w_{32}\left(0, y_{3}\right), \quad y_{3}=L_{3}-\lambda_{32} t$.

Case B When the outgoing characteristic at the free endpoint, starts from $M$ (strong lines in Fig. 3). 
Fig. 3 The characteristics at the endpoints of the network and their starting points. For a time $t$ such that $t \leq \frac{L_{1}}{\left|\lambda_{11}\right|}$ for canal 1 , $t \leq \frac{L_{2}}{\lambda_{22}}$ for canal 2 and $t \leq \frac{L_{3}}{\lambda_{32}}$ for canal 3, the starting points are within the corresponding canals. Otherwise, the starting points are at the junction $M$

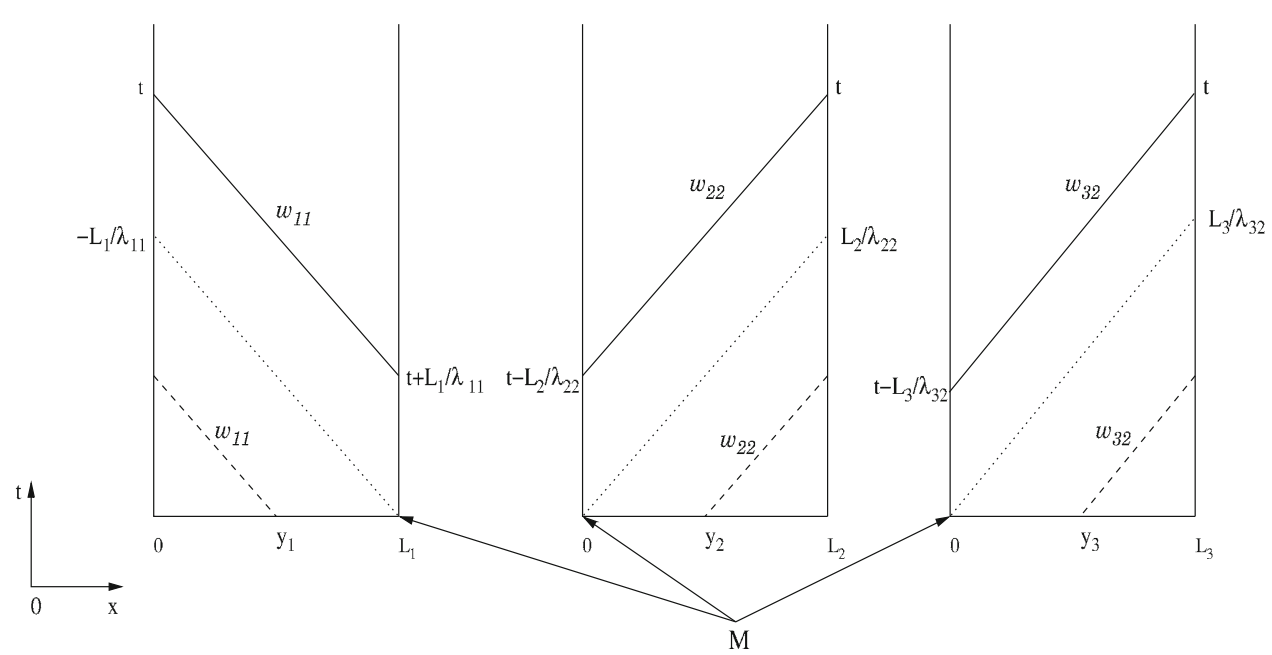

This case involves the junction conditions and occurs when $t \geq \frac{L_{1}}{\left|\lambda_{11}\right|}$ for canal $1, t \geq \frac{L_{2}}{\lambda_{22}}$ for canal 2 and $t \geq \frac{L_{3}}{\lambda_{32}}$ for canal 3. Using (14), the following relations hold

$$
\begin{aligned}
& w_{11}(t, 0)=w_{11}\left(t-\frac{L_{1}}{\left|\lambda_{11}\right|}, L_{1}\right), \\
& w_{22}\left(t, L_{2}\right)=w_{22}\left(t-\frac{L_{2}}{\lambda_{22}}, 0\right), \\
& w_{32}\left(t, L_{3}\right)=w_{32}\left(t-\frac{L_{3}}{\lambda_{32}}, 0\right) .
\end{aligned}
$$

Using (17) one has

$\left(\begin{array}{c}w_{11}\left(t, L_{1}\right) \\ w_{22}(t, 0) \\ w_{32}(t, 0)\end{array}\right)=D\left(\begin{array}{c}w_{12}\left(t, L_{1}\right) \\ w_{21}(t, 0) \\ w_{31}(t, 0)\end{array}\right)$,

where $D=\left(d_{i j}\right)_{1 \leq i, j \leq 3}=B^{-1} C$. Hence, from (67-69), we get

$\left(\begin{array}{c}w_{11}\left(t+L_{1} /\left|\lambda_{11}\right|, 0\right) \\ w_{22}\left(t+L_{2} / \lambda_{22}, L_{2}\right) \\ w_{32}\left(t+L_{3} / \lambda_{32}, L_{3}\right)\end{array}\right)=D\left(\begin{array}{c}w_{12}\left(t, L_{1}\right) \\ w_{21}(t, 0) \\ w_{31}(t, 0)\end{array}\right)$.

Note that one can derive relations similar to (64-66) and (67-69) for $w_{12}, w_{21}$ and $w_{31}$ at the junction $M$, i.e:

$$
\begin{aligned}
& w_{12}\left(t, L_{1}\right)= \begin{cases}w_{12}\left(0, L_{1}-\lambda_{12} t\right), & t \in\left[0, \frac{L_{1}}{\lambda_{12}}\right], \\
w_{12}\left(t-\frac{L_{1}}{\lambda_{12}}, 0\right), & t \geq \frac{L_{1}}{\lambda_{12}},\end{cases} \\
& w_{21}(t, 0)= \begin{cases}w_{21}\left(0,\left|\lambda_{21}\right| t\right), & t \in\left[0, \frac{L_{2}}{\left.\mid \lambda_{21}\right]}\right], \\
w_{21}\left(t-\frac{L_{2}}{\left|\lambda_{21}\right|}, L_{2}\right), & t \geq \frac{L_{2}}{\left|\lambda_{21}\right|},\end{cases}
\end{aligned}
$$

and

$$
w_{31}(t, 0)= \begin{cases}w_{31}\left(0,\left|\lambda_{31}\right| t\right), & t \in\left[0, \frac{L_{3}}{\left|\lambda_{31}\right|}\right], \\ w_{31}\left(t-\frac{L_{3}}{\left|\lambda_{31}\right|}, L_{3}\right), & t \geq \frac{L_{3}}{\left|\lambda_{31}\right|} .\end{cases}
$$

We denote

$$
b_{1}(t)=\left\{\begin{array}{l}
w_{11}\left(0,\left|\lambda_{11}\right| t\right), \quad t \leq \frac{L_{1}}{\left|\lambda_{11}\right|}, \\
d_{11} w_{12}\left(t-\frac{L_{1}}{\left|\lambda_{11}\right|}, L_{1}\right)+d_{12} w_{21}\left(t-\frac{L_{1}}{\left|\lambda_{11}\right|}, 0\right) \\
+d_{13} w_{31}\left(t-\frac{L_{1}}{\left|\lambda_{11}\right|}, 0\right), \quad t \geq \frac{L_{1}}{\left|\lambda_{11}\right|} .
\end{array}\right.
$$

$b_{2}(t)=\left\{\begin{array}{l}w_{22}\left(0, L_{2}-\lambda_{22} t\right), \quad t \leq \frac{L_{2}}{\lambda_{22}}, \\ d_{21} w_{12}\left(t-\frac{L_{2}}{\lambda_{22}}, L_{1}\right)+d_{22} w_{21}\left(t-\frac{L_{2}}{\lambda_{22}}, 0\right) \\ +d_{23} w_{31}\left(t-\frac{L_{2}}{\lambda_{22}}, 0\right), \quad t \geq \frac{L_{2}}{\lambda_{22}} .\end{array}\right.$

$b_{2}(t)=\left\{\begin{array}{l}w_{32}\left(0, L_{3}-\lambda_{32} t\right), \quad t \leq \frac{L_{3}}{\lambda_{32}}, \\ d_{31} w_{12}\left(t-\frac{L_{3}}{\lambda_{32}}, L_{1}\right)+d_{32} w_{21}\left(t-\frac{L_{3}}{\lambda_{32}}, 0\right) \\ +d_{33} w_{31}\left(t \frac{L_{3}}{\lambda_{32}}, 0\right), \quad t \geq \frac{L_{3}}{\lambda_{32}} .\end{array}\right.$

Therefore we have proved the Lemma 1.

From (13), one deduces that

$\hat{h}_{1}(t, 0)=\frac{1}{l_{1} \lambda_{12}} \hat{q}_{1,0}(t)+\frac{1}{l_{1}} w_{11}(t, 0)$,

$\hat{h}_{2}\left(t, L_{2}\right)=\frac{1}{l_{2} \lambda_{21}} \hat{q}_{2, L_{2}}(t)+\frac{1}{l_{2}} w_{22}\left(t, L_{2}\right)$,

$\hat{h}_{3}\left(t, L_{3}\right)=\frac{1}{l_{3} \lambda_{31}} \hat{q}_{3, L_{3}}(t)+\frac{1}{l_{3}} w_{32}\left(t, L_{3}\right)$.

Therefore, we deduce from (63) and Lemma 1 that

$$
\begin{gathered}
\frac{1}{2} \frac{\partial}{\partial t} E(t)=a_{1} \hat{q}_{1,0}^{2}(t)+\frac{\gamma_{1}}{l_{1}} b_{1}(t) \hat{q}_{1,0}(t)+a_{2} \hat{q}_{2, L_{2}}^{2}(t) \\
-\frac{\gamma_{2}}{l_{2}} b_{2}(t) \hat{q}_{2, L_{2}}(t)+a_{3} \hat{q}_{3, L_{3}}^{2}(t)-\frac{\gamma_{3}}{l_{3}} b_{3}(t) \hat{q}_{3, L_{3}}(t),
\end{gathered}
$$

where $a_{i}=\frac{\delta_{i}}{2}\left(\lambda_{i 2}+\left|\lambda_{i 1}\right|\right)>0$ depends only on the steady state and $\gamma_{i}$ are defined in (12). One chooses the controllers $\hat{q}_{2, L_{2}}, \hat{q}_{3, L_{3}}$ and $\hat{q}_{1,0}$ as in (21). 
By applying Lemma 2 to (21), we have

$$
\begin{aligned}
\frac{1}{2} \frac{d}{d t} E(t)= & -\frac{\left(\gamma_{1} b_{1}(t)\right)^{2}}{4 l_{1}^{2} a_{1}} \theta_{1}(t)-\frac{\left(\gamma_{2} b_{2}(t)\right)^{2}}{4 l_{2}^{2} a_{2}} \theta_{2}(t) \\
& -\frac{\left(\gamma_{3} b_{3}(t)\right)^{2}}{4 l_{3}^{2} a_{3}} \theta_{3}(t) .
\end{aligned}
$$

\subsection{Stabilization process}

The following Theorem deals with smooth data while Theorem 1 concerns data with less regularity. Besides, the proof of Theorem 1 will be based on the following Theorem (see Sect. 5.4).

Theorem 4 Let $t_{k}=k T_{0}$ where $T_{0}$ is given by (22). Assume that (16) holds, the initial condition $\left(\hat{h}_{i}^{0}, \hat{q}_{i}^{0}\right)$ is continuous in $\left[0, L_{i}\right], \theta_{i}(i=1,2,3)$ are continuous in $\left.] 0,1\right]$ and ( $\left.\hat{q}_{1,0}, \hat{q}_{2, L_{2}}, \hat{q}_{3, L_{3}}\right)$ satisfies (21). Then (9) has a unique solution $\left(\hat{h}_{i}, \hat{q}_{i}\right)_{i=1,2,3}$ continuous in $\left[t_{k}, t_{k+1}\right] \times\left[0, L_{i}\right]$ such that the energy is non-increasing in $\left[t_{k}, t_{k+1}\right]$ and satisfies:

$E\left(t_{k+1}\right) \leq\left(1-\Theta^{k}\right) E\left(t_{k}\right)$.

Here, $\left.\Theta^{k}=\min \left(\Delta_{1}^{k}, \Delta_{2}^{k}, \Delta_{3}^{k}\right) \in\right] 0,1[$,

$\Delta_{1}^{k}=\min \left(\frac{\left|\lambda_{11}\right|}{\lambda_{12}+\left|\lambda_{11}\right|} \inf _{x \in\left[0, L_{1}\right]} \theta_{1}\left(t_{k}+\frac{x}{\left|\lambda_{11}\right|}\right), \frac{\gamma_{1} \epsilon}{l_{1}}\right)$,

$\Delta_{2}^{k}=\min \left(\frac{\left|\lambda_{21}\right|}{\lambda_{22}+\left|\lambda_{21}\right|} \inf _{x \in\left[0, L_{2}\right]} \theta_{2}\left(t_{k}+\frac{L_{2}-x}{\lambda_{22}}\right), \frac{\gamma_{2} \epsilon}{l_{2}}\right)$,

$\Delta_{3}^{k}=\min \left(\frac{\left|\lambda_{31}\right|}{\lambda_{32}+\left|\lambda_{31}\right|} \inf _{x \in\left[0, L_{3}\right]} \theta_{3}\left(t_{k}+\frac{L_{3}-x}{\lambda_{32}}\right), \frac{\gamma_{3} \epsilon}{l_{3}}\right)$,

and $\epsilon>0$ is given by equivalence of norms (see the Appendix).

The proof of Theorem 4 is given in the Appendix.

\subsection{Proof of Theorem 1}

We argue as for the proof of Theorem 3. One considers subsequences $\left(\hat{h}_{i, n}\right)_{n \geq 0} \subset C^{0}\left(\left[0, L_{i}\right]\right)$ and $\left(\hat{q}_{i, n}\right)_{n \geq 0} \subset$ $C^{0}\left(\left[0, L_{i}\right]\right)$ such that

$\hat{h}_{i, n}^{0} \longrightarrow \hat{h}_{i}^{0}$ and $\hat{q}_{i, n}^{0} \longrightarrow \hat{q}_{i}^{0}$ in $L^{2}\left(\left[0, L_{i}\right]\right), \quad n \longrightarrow+\infty$.

This is possible, because $\left(\hat{h}_{i}^{0}, \hat{q}_{i}^{0}\right) \in\left(L^{2}\left(\left[0, L_{i}\right]\right)\right)^{2}$.

For any $n \geq 0$, we define $\hat{q}_{1,0, n}(t), \hat{q}_{2, L_{2}, n}(t)$ and $\hat{q}_{3, L_{3, n}, n}(t)$ as in (21), where $\hat{q}_{i}$ and $\hat{h}_{i}$ are replaced by $\hat{q}_{i, n}$ and $\hat{h}_{i, n}$ respectively. We consider that the subsequences satisfy the weak formulation (60-61).

By using Theorem 4, the energy

$E^{n}(t)=\sum_{i=1}^{3}\left(\left\|\sqrt{l_{i} \gamma_{i}} \hat{h}_{i, n}(t)\right\|_{2, \Omega}^{2}+\left\|\sqrt{\delta_{i}} \hat{q}_{i, n}(t)\right\|_{2, \Omega}^{2}\right)$ satisfies (83) and can be written as (56) which can be combined with $(9 \mathrm{a}-\mathrm{b})$ to get that

$$
\left(\begin{array}{c}
l_{i} \hat{h}_{i, n} \\
\hat{q}_{i, n}
\end{array}\right), \quad\left(\begin{array}{c}
\hat{q}_{i, n} \\
\sigma \hat{q}_{i, n}+\rho \hat{h}_{i, n}
\end{array}\right) \in H\left(\operatorname{div}, Q_{i}\right) .
$$

and bounded in $H\left(\operatorname{div}, Q_{i}\right)$. For $T>0$, using the Banach reflexive space $L^{2}\left(0, T ; L^{2}(\Omega)^{2}\right)$ and [28], one can show that there exists subsequences weakly converging to $\left(\hat{h}_{i}, \hat{q}_{i}\right)$ in $L^{2}\left(0, T ; L^{2}\left(\Omega_{i}\right)^{2}\right)$. Moreover, $\left(\hat{h}_{i}, \hat{q}_{i}\right)$ satisfies $(9-11)$.

\section{Numerical results}

In this section, we present numerical results. First, we consider the case of a single canal and afterward we treat the case of the network. In both cases, a high order finite volume method is used to solve numerically the nonlinear model. The method is based on subdividing the spatial domain into grid cells and approximating the integral of $(h, q)^{t}$ over these cells by solving Riemann problem at interfaces between cells. For the procedure of solving numerically the nonlinear SaintVenant equations, one can refer to [25] and [26]. At the junction, a Roe linearisation has been used to define characteristic variables. An extrapolation based on Upwind methods is used for incoming characteristic variables at $M$. The outgoing characteristic variables are derived from the junction conditions (4-5).

We have considered a spatial step size $\Delta x=0.5 \mathrm{~m}$ and a time step $\Delta t=1 \mathrm{~s}$. Also different choice of spatial step size have shown that the solution of the numerical method converges.

Note that the controllers are built through the linear system but in this section they are applied to the nonlinear shallow water equations. The numerical experiments show that these controllers lead to stability of the nonlinear problem even when big perturbations are considered.

\subsection{Numerical results for a single canal}

For the simulation, we illustrate the control design method on a single canal of Length $L=50 \mathrm{~m}$, width $l=1 \mathrm{~m}$. The steady state is $\bar{q}(x)=1.5 \mathrm{~m}^{3} \mathrm{~s}^{-1}$ and $\bar{h}(x)=1 \mathrm{~m}$ and the initial condition is $h(0, x)=2 \mathrm{~m}$ and $q(0, x)=2.5 \mathrm{~m}^{3} \mathrm{~s}^{-1}$.

The boundary conditions are

$q(t, 0)=\bar{q}(x)+\hat{q}_{0}(t), \quad q(t, L)=\bar{q}(x)+\hat{q}_{L}(t)$,

where $\hat{q}_{0}(t)$ and $\hat{q}_{L}(t)$ are the time-delay feedback controllers given by (47).

The initial condition is constant in space. Initially, waves are generated from the two endpoints. As they reach the opposite endpoint they are partially reflected.

One can see from Fig. 4, that the bigger the $\theta$ 's are, the faster the exponential decrease is. 


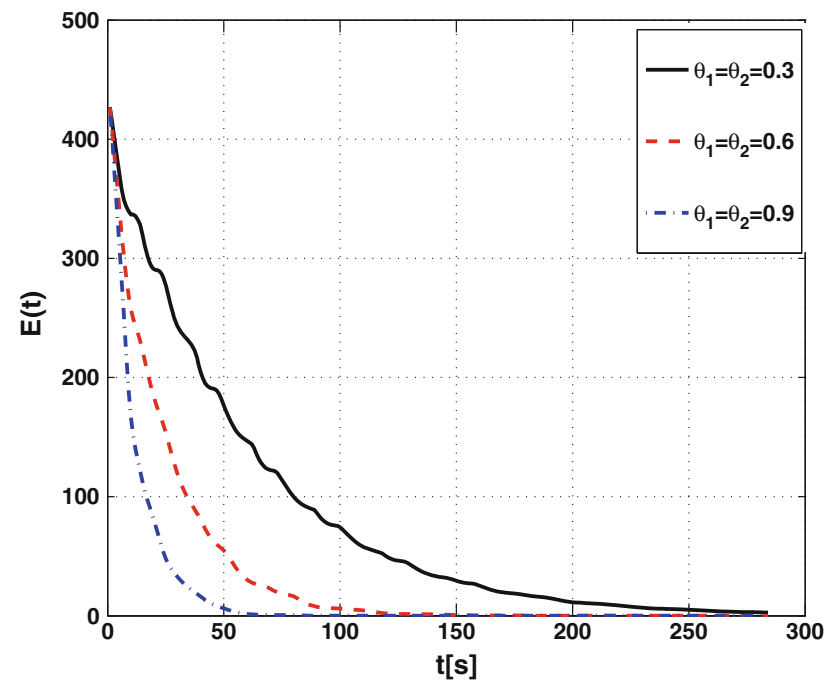

Fig. 4 Energy evolution of the single canal for different values of $\theta_{1}$ and $\theta_{2}$

6.2 Numerical results for the network

For a simulation example,

- we consider a network where $L_{1}=30 \mathrm{~m}, L_{2}=40 \mathrm{~m}$, $L_{3}=50 \mathrm{~m}$ and $l_{1}=l_{2}=l_{3}=1 \mathrm{~m}$.

- For the steady state, we give $\bar{q}_{1,0}=1.5 \mathrm{~m}^{3} \mathrm{~s}^{-1}, \bar{q}_{2, L_{2}}=$ $1 \mathrm{~m}^{3} \mathrm{~s}^{-1}, \bar{h}_{3, L_{3}}=1 \mathrm{~m}$ and derive $\left(\bar{h}_{1}(x), \bar{q}_{1}(x)\right),\left(\bar{h}_{2}(x)\right.$, $\left.\bar{q}_{2}(x)\right)$ and $\left(\bar{h}_{3}(x), \bar{q}_{3}(x)\right)$ by solving the steady state system (7).

- The initial condition is $\left(h_{1}^{0}(x), q_{1}^{0}(x)\right)=\left(2 \mathrm{~m}, 2.5 \mathrm{~m}^{3} \mathrm{~s}^{-1}\right)$ for canal $1,\left(h_{2}^{0}(x), q_{2}^{0}(x)\right)=\left(h_{2}^{0}(0), 1.5 \mathrm{~m}^{3} \mathrm{~s}^{-1}\right)$ for canal 2 and $\left(h_{3}^{0}(x), q_{3}^{0}(x)\right)=\left(h_{3}^{0}(0), q_{3}^{0}(0)\right)$ for canal 3. Here, $h_{2}^{0}(0), q_{3}^{0}(0)$ and $h_{3}^{0}(0)$ are derived by (4) and (5), respectively.

The boundary conditions are

$q_{1}(t, 0)=\bar{q}_{1}(x)+\hat{q}_{1,0}(t)$,

$q_{2}\left(t, L_{2}\right)=\bar{q}_{2}(x)+\hat{q}_{2, L_{2}}(t)$,

$q_{3}\left(t, L_{3}\right)=\bar{q}_{3}(x)+\hat{q}_{3, L_{3}}(t)$,

where $\hat{q}_{1,0}, \hat{q}_{2, L_{2}}$ and $\hat{q}_{3, L_{3}}$ are the time-delay feedback controllers given by (21).

The initial condition is constant in each canal. Initially wave are generated from the outer endpoints and from the junction. When the waves reach the outer endpoints they are partially reflected, and when waves reach the junction there is transmissions and reflection. A very complicated wave pattern emerges (Fig. 5 ).

\subsection{Discussion}

For the single canal and the network, note that we have considered big perturbations, in order to investigate the robust-

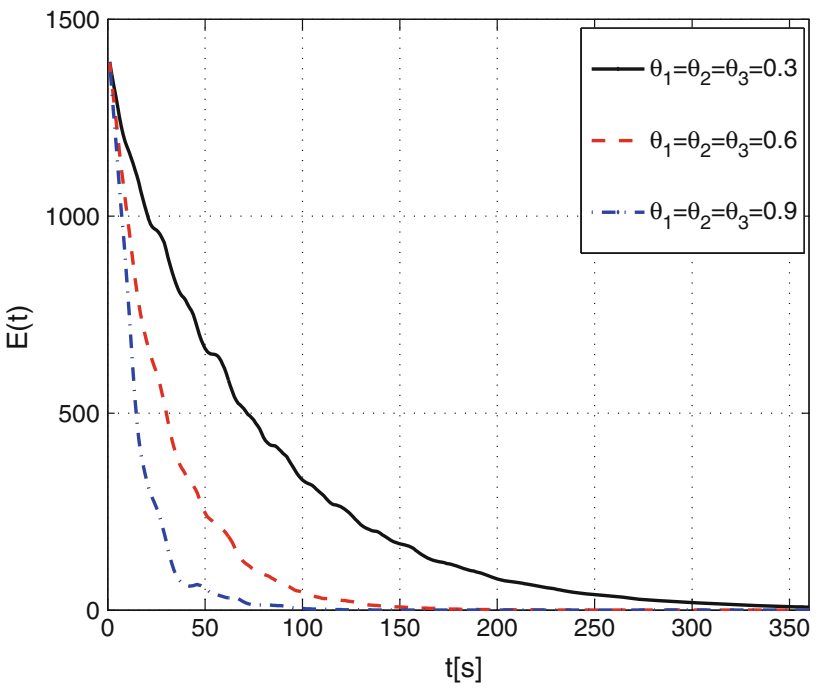

Fig. 5 Evolution of the energy of the network for different values of $\theta_{1}, \theta_{2}$ and $\theta_{3}$

ness and the flexibility of the control method. We have also tested smaller perturbations with the same theta functions. In all cases, one has noticed almost the same shape of the energy decay. The magnitude of the control depends on the magnitude of the perturbations and on the choice of the $\theta$ 's. More computations, with different perturbations, have been performed and in all cases the controllers have permitted to get the steady state.

Acknowledgments This work has been supported by ISP (International Science Program), Sweden and Aires-Sud Project-France, UMMISCO-IRD Project-France and Fond National Suisse.

\section{Appendix}

Proof [of Theorem 4] Integrating (82) from 0 to $t_{1}$ one has

$$
\begin{aligned}
E\left(t_{1}\right) \leq & E(0)-\int_{0}^{t_{1}}-\frac{\left(\gamma_{1} b_{1}(t)\right)^{2}}{4 l_{1}^{2} a_{1}} \theta_{1}(t) d t \\
& -\int_{0}^{t_{1}} \frac{\left(\gamma_{2} b_{2}(t)\right)^{2}}{4 l_{2}^{2} a_{2}} \theta_{2}(t) d t-\int_{0}^{t_{1}} \frac{\left(\gamma_{3} b_{3}(t)\right)^{2}}{4 l_{3}^{2} a_{3}} \theta_{3}(t) d t,
\end{aligned}
$$

Then, by using $b_{i}$ defined in Lemma 1 and changing the integrations variables, one has

$$
\begin{aligned}
& E\left(t_{1}\right) \leq E(0)-\frac{\gamma_{1}^{2}}{2 l_{1}^{2} a_{1}\left|\lambda_{11}\right|} \int_{0}^{L_{1}} w_{11}^{2}(0, x) \theta_{1}\left(\frac{x}{\left|\lambda_{11}\right|}\right) d x \\
& -\frac{\gamma_{2}^{2}}{2 l_{2}^{2} a_{2} \lambda_{22}} \int_{0}^{L_{2}} w_{22}^{2}(0, x) \theta_{2}\left(\frac{L_{2}-x}{\lambda_{22}}\right) d x
\end{aligned}
$$




$$
\begin{aligned}
& -\frac{\gamma_{3}^{2}}{2 l_{3}^{2} a_{3} \lambda_{32}} \int_{0}^{L_{3}} w_{32}^{2}(0, x) \theta_{3}\left(\frac{L_{3}-x}{\lambda_{32}}\right) d x \\
& -\frac{\gamma_{1}^{2}}{2 l_{1}^{2} a_{1}} \int_{0}^{\tau_{1}}\left[d_{11} w_{12}\left(s, L_{1}\right)+\xi_{1}(s, 0)\right]^{2} \theta_{1}\left(s+\frac{L_{1}}{\left|\lambda_{11}\right|}\right) d s \\
& -\frac{\gamma_{2}^{2}}{2 l_{2}^{2} a_{2}} \int_{0}^{\tau_{2}}\left[d_{21} w_{12}\left(s, L_{1}\right)+\xi_{2}(s, 0)\right]^{2} \theta_{2}\left(s+\frac{L_{2}}{\lambda_{22}}\right) d s \\
& -\frac{\gamma_{3}^{2}}{2 l_{3}^{2} a_{3}} \int_{0}^{\tau_{3}}\left[d_{31} w_{12}\left(s, L_{1}\right)+\xi_{3}(s, 0)\right]^{2} \theta_{3}\left(s+\frac{L_{3}}{\lambda_{32}}\right) d s .
\end{aligned}
$$

where $\xi_{i}(s, 0)=d_{i 2} w_{21}(s, 0)+d_{i 3} w_{31}(s, 0), \tau_{1}=t_{1}-\frac{L_{i}}{\left|\lambda_{i j}\right|}$, $\tau_{2}=t_{1}-\frac{L_{2}}{\lambda_{22}}$ and $\tau_{3}=t_{1}-\frac{L_{3}}{\lambda_{32}}$.

Let us define define $\Gamma_{i}^{j}(i=1, \ldots, 3, j=0,1)$ as follows

$$
\begin{aligned}
& \Gamma_{1}^{0}=\frac{\gamma_{1}^{2}}{2 l_{1}^{2} a_{1}\left|\lambda_{11}\right|} \inf _{x \in\left[0, L_{1}\right]} \theta_{1}\left(\frac{x}{\left|\lambda_{11}\right|}\right), \\
& \Gamma_{2}^{0}=\frac{\gamma_{2}^{2}}{2 l_{2}^{2} a_{2} \lambda_{22}} \inf _{x \in\left[0, L_{2}\right]} \theta_{2}\left(\frac{L_{2}-x}{\lambda_{22}}\right), \\
& \Gamma_{3}^{0}=\frac{\gamma_{3}^{2}}{2 l_{3}^{2} a_{3} \lambda_{32}} \inf _{x \in\left[0, L_{3}\right]} \theta_{3}\left(\frac{L_{3}-x}{\lambda_{32}}\right), \\
& \Gamma_{1}^{1}=\frac{\gamma_{1}^{2}}{2 l_{1}^{2} a_{1}} \inf _{s \in\left[0, t_{1}-\frac{L_{1}}{\left|\lambda_{11}\right|}\right]} \theta_{1}\left(s+\frac{L_{1}}{\left|\lambda_{11}\right|}\right), \\
& \Gamma_{2}^{1}=\frac{\gamma_{2}^{2}}{2 l_{2}^{2} a_{2}} \inf _{s \in\left[0, t_{1}-\frac{L_{2}}{\lambda_{22}}\right]} \theta_{2}\left(s+\frac{L_{2}}{\lambda_{22}}\right), \\
& \Gamma_{3}^{1}=\frac{\gamma_{3}^{2}}{2 l_{3}^{2} a_{3}} \inf _{s \in\left[0, t_{1}-\frac{L_{3}}{\lambda_{32}}\right]} \theta_{3}\left(s+\frac{L_{3}}{\lambda_{32}}\right) .
\end{aligned}
$$

Then, from (86) is deduced the following inequality:

$$
\begin{aligned}
& E\left(t_{1}\right) \leq E(0)-\Gamma_{1}^{0} \int_{0}^{L_{1}} w_{11}^{2}(0, x) d x \\
& -\Gamma_{2}^{0} \int_{0}^{L_{2}} w_{22}^{2}(0, x) d x-\Gamma_{3}^{0} \int_{0}^{L_{3}} w_{32}^{2}(0, x) d x \\
& -\Gamma_{1}^{1} \int_{0}^{\tau_{1}}\left(d_{11} w_{12}\left(s, L_{1}\right)+\xi_{1}(s, 0)\right)^{2} d s \\
& -\Gamma_{2}^{1} \int_{0}^{\tau_{2}}\left(d_{21} w_{12}\left(s, L_{1}\right)+\xi_{2}(s, 0)\right)^{2} d s \\
& -\Gamma_{3}^{1} \int_{0}^{\tau_{3}}\left(d_{31} w_{12}\left(s, L_{1}\right)+\xi_{3}(s, 0)\right)^{2} d s .
\end{aligned}
$$

Since $\min \left(\tau_{1}, \tau_{2}, \tau_{3}\right) \geq \tau_{0}=\max \left(\frac{L_{1}}{\lambda_{12}}, \frac{L_{2}}{\left|\lambda_{21}\right|}, \frac{L_{3}}{\left|\lambda_{31}\right|}\right)$, inequality (86) implies

$$
\begin{aligned}
E\left(t_{1}\right) \leq & E(0)-\int_{0}^{\tau_{0}}\|A D X\|^{2} d s-\Gamma_{1}^{0} \int_{0}^{L_{1}} w_{11}^{2}(0, x) d x \\
& -\Gamma_{2}^{0} \int_{0}^{L_{2}} w_{22}^{2}(0, x) d x-\Gamma_{3}^{0} \int_{0}^{L_{3}} w_{32}^{2}(0, x) d x
\end{aligned}
$$

here $D$ is given in (70) and

$$
A=\left(\begin{array}{ccc}
\Gamma_{1}^{1} & 0 & 0 \\
0 & \Gamma_{2}^{1} & 0 \\
0 & 0 & \Gamma_{3}^{1}
\end{array}\right), \quad X=\left(\begin{array}{c}
w_{12}\left(s, L_{1}\right) \\
w_{21}(s, 0) \\
w_{31}(s, 0)
\end{array}\right)
$$

Since matrix $A D$ is invertible, there exists $\epsilon>0$ such that $-\|A D X\|^{2} \leq-\epsilon\|X\|^{2}$. Then, by considering identities (72-74) and estimation (87) one gets

$$
\begin{aligned}
E\left(t_{1}\right) \leq & E(0)-\Gamma_{1}^{0} \int_{0}^{L_{1}} w_{11}^{2}(0, x) d x-\Gamma_{2}^{0} \int_{0}^{L_{2}} w_{22}^{2}(0, x) d x \\
& -\Gamma_{3}^{0} \int_{0}^{L_{3}} w_{32}^{2}(0, x) d x-\epsilon \int_{0}^{\frac{L_{1}}{\lambda_{12}}} w_{12}^{2}\left(0, L_{1}-\lambda_{12} s\right) d s \\
& -\epsilon \int_{0}^{\frac{L_{2}}{\left|\lambda_{21}\right|}} w_{21}^{2}\left(0,\left|\lambda_{21}\right| s\right) d s-\epsilon \int_{0}^{\frac{L_{3}}{\left|\lambda_{31}\right|}} w_{31}^{2}\left(0,\left|\lambda_{31}\right| s\right) d s .
\end{aligned}
$$

Now, we take a change of variables to get

$$
\begin{aligned}
E\left(t_{1}\right) \leq & E(0)-\Pi_{1}^{0} \int_{0}^{L_{1}}\left[w_{11}^{2}(0, x)+w_{12}^{2}(0, x)\right] d x \\
& -\Pi_{2}^{0} \int_{0}^{L_{2}}\left[w_{22}^{2}(0, x)+w_{21}^{2}(0, x)\right] d x \\
& -\Pi_{3}^{0} \int_{0}^{L_{3}}\left[w_{32}^{2}(0, x)+w_{31}^{2}(0, x)\right] d x .
\end{aligned}
$$

where $\Pi_{1}^{0}=\min \left(\Gamma_{1}^{0}, \frac{\epsilon}{\lambda_{12}}\right), \Pi_{2}^{0}=\min \left(\Gamma_{2}^{0}, \frac{\epsilon}{\left|\lambda_{21}\right|}\right)$ and $\Pi_{3}^{0}=\min \left(\Gamma_{3}^{0}, \frac{\epsilon}{\left|\lambda_{31}\right|}\right)$.

Let us recall that (51), gives the following estimation $w_{i 2}^{2}(0, x)+w_{i 1}^{2}(0, x) \geq \frac{l_{i}}{\gamma_{i}}\left(l_{i} \gamma_{i}\left(\hat{h}_{i}^{0}(x)\right)^{2}+\delta_{i}\left(\hat{q}_{i}^{0}(x)\right)^{2}\right)$. 
Let us denote $\Delta_{i}^{0}=\frac{l_{i} \Pi_{i}^{0}}{\gamma_{i}}$, then by combining (88) and (89), one gets

$$
\begin{aligned}
E\left(t_{1}\right) & \leq E(0)-\left(\Delta_{1}^{0} E_{1}(0)+\Delta_{2}^{0} E_{2}(0)+\Delta_{3}^{0} E_{3}(0)\right) \\
& \leq\left(1-\Theta^{0}\right) E(0),
\end{aligned}
$$

where

$\Theta^{0}=\min \left(\Delta_{1}^{0}, \Delta_{2}^{0}, \Delta_{3}^{0}\right)$.

On the other hand, we have

$\Delta_{2}^{0}=\min \left(\frac{\left|\lambda_{21}\right|}{\lambda_{22}+\left|\lambda_{21}\right|} \inf _{x \in\left[0, L_{2}\right]} \theta_{2}\left(\frac{L_{2}-x}{\lambda_{22}}\right), \frac{\gamma_{2} \epsilon}{l_{2} \lambda_{22}}\right)<1$.

Therefore $\Theta^{0}<1$.

To generalize with respect to time, we consider $t_{k}$ as initial time and argue as for the proof of Theorem 2. Then, the proof of Theorem 4 is complete.

\section{References}

1. de SAINT-VENANT B (1871) Théorie du mouvement nonpermanent des eaux avec application aux crues des riviéres et á l'introduction des marées dans leur lit. C R Acad Sci 73(148154):237-240

2. Russel DL (1978) Controllability and stabilizability theory for linear partial differential equations: recent progress and open questions. SIAM Rev 20(4):639-739

3. Malaterre PO, Roge DC (1998) Classification of canal control algorithms. J Irrig Drain Eng 124(1):3-10

4. Bastin G, Bayen AM, D'apice C, Litrico X, Piccoli B (2009) Open problems and research perspectives for irrigation channels. Netw Heterog Media 4(2):1-4

5. Balogun O, Hubbard M, De Vries JJ (1988) Automatic control of canal flow using linear quadratic regulator theory. J Hydraul Eng 114(1):75-102

6. Malaterre PO (1998) PILOTE linear quadratic optimal controller for irrigation canals. J Irrig Drain Eng 124(4):187-193

7. Weyer E (2003) LQ control of irrigation channel. Decis Control 1:750-755

8. Chen M, Georges D, Lefevre L (2002) Infinite dimensional LQ control of an open-channel hydraulic system. ASCC, Singapore

9. Xu CZ, Sallet G (1999) Proportional and integral regulation of irrigation canal systems governed by the Saint-Venant equation. In: Proceedings of the 14th world congress IFAC. Elsevier, Beijing, pp $147-152$
10. Litrico X, Vincent $F(2006) H_{\infty}$ control of an irrigation canal pool with a mixed control politics. IEEE Trans Autom Control Syst Technol 14(1):99-111

11. Gugat M, Dick M (2011) Time-delayed boundary feedback stabilization of the isothermal Euler equations with friction. Math Control Relat Fields 1(4):469-491

12. Leugering G, Georg Schmidt EJP (2002) On the modelling and stabilization of flows in networks of open canal. SIAM J Control Optim 41(1):164-180

13. Gugat M, Leugering G, Georg Schmidt EJP (2004) Global controllability between steady supercritical flows in channel networks. Math Methods Appl Sci 27:781-802

14. de Halleux J, Prieur C, Coron J-M, d'Andréa-Novel B, Bastin G (2003) Boundary feedback control in networks of open channels. Automatica 39(8):1365-1376

15. Ndiaye M, Bastin G (2004) Commande fontiere adptative d'un bief de canal avec prélévements inconnus. RS-JESA 38:374-371

16. Cen L.-H., Xi Y.-G. (2009) Stability of boundary feedback control based on weighted Lyapunov function in networks of open channels. Acta Autom Sin 35(1):97-102

17. Bastin G, Coron JM, D’Andréa-Novel B (2009) On Lyapunov stability of linearised Saint-Venant equations for a sloping channel. Netw Heterog Media 4(2):177-187

18. Séne A, Wane BA, Le Roux DY (2008) Control of irrigation channels with variable bathymetry and time dependent stabilization rate. C R Acad Sci Paris I 346:1119-1122

19. Misra R, Mohan Kumar MS, Sridharan K (1991) Analysis of transients in a canal network. Sādhanā 16(1):85-89

20. X. Litrico (2001) Robust flow control of single input multiple outputs regulated rivers. J Irrig Drain Eng 127(5):281-286

21. Li T (2005) Exact boundary controlability of unsteady flows in a network of open canals. Math Nachr 278(3):278-289

22. Qilong G, Li T (2008) Exact boundary observability of unsteady flows in a tree-like network of open canals. Math Methods Appl Sci. doi:10.1002/mma.1043

23. Qilong G (2008) Exact boundary controllability of unsteady supercritical flows in a tree-like network of open canals. Math Methods Appl Sci 31:1497-1508

24. Gugat M, Dick M, Leugering G (2013) Stabilization of the gas flow in star-shaped networks by feedback controls with varying delay. Syst Model Optim IFIP Adv Inf Commun Technol 391(4):255-265

25. Leveque RJ (2002) Finite volume methods for hyperbolic problems. Cambridge texts in applied mathematics. Cambridge University Press, Cambridge

26. Toro EF (1999) Riemann solvers and numerical methods for fluid dynamics: a practical introduction. Springer, Berlin

27. Dos Santos V, Bastin G, Coron JM, D'Andréa-Novel B (2003) Boundary control with integral action for hyperbolic systems of conservation laws: stability and experiments. Automatica 44:1310 1318

28. Girault V, Raviart PA (1986) Finite elements methods for NavierStokes equations. Theory and algorithms. Springer, Berlin 